\title{
Rapport général de la question II « Rives, rivières, riverains et écosystèmes aquatiques »
}

\author{
par J.-L. Beseme et F. Nioche-Seigneuret \\ Agence de l'Eau Loire-Bretagne
}

\section{INTRODUCTION}

L'eau, au centre de nos préoccupations durant ce congrès, doit être gérée et protégée au mieux de nos possibilités parce qu'elle occupe une place importante sur l'échelle des valeurs de nos ressources naturelles, et qu'elle constitue un des facteurs de développement de toute société : l'évolution des techniques, ces dernières décennies, a contribué à la mise en place d'actions curatives importantes, mais aussi à la «maltraitance " du réseau hydrographique et des milieux connexes. Il a fallu un certain temps pour que l'on accorde aux cours d'eau et à leurs espaces associés l'attention dont ils ont besoin, dans un contexte interdisciplinaire. Les progrès à accomplir pour préserver les hydrosystèmes fluviaux, caractérisés par leurs richesses biologiques et leurs activités économiques, se résument à deux niveaux d'intervention :

- le bassin versant ou approche globale destinée aux choix d'aménagement de l'espace (stratégie de gestion des débits, de dépollution, de développement économique),

- l'hydrosystème ou approche locale destinée aux petits aménagements, à la gestion quotidienne, et dont les choix s'insèrent dans la stratégie globale définie au niveau précédent.

L'interdépendance bassin versant-hydrosystème réside en l'alimentation par gravité du réseau hydrographique de surface et des réserves en eaux souterraines. Elle s'illustre par l'impact de l'évolution de l'occupation des sols du bassin versant sur les milieux aquatiques (incidences sur les crues, le fonctionnement physique, les habitats, la qualité des eaux, les ressources en eau...).

La question 2, par son intitulé : « Rives, rivières, riverains et écosystèmes aquatiques » cible :

- des milieux : l'hydrosystème constitué du cours d'eau, de ses lits et de sa vallée,

- des hommes : les riverains et les usages qu'ils sous-tendent.

Les uns et les autres ont des besoins compatibles dans certains cas, contradictoires dans d'autres. Comment envisager une cohabitation sereine entre écosystème et sociosystème alors que ce dernier s'est imposé par le passé, parfois au détriment du premier?
Le contexte réglementaire européen et national laisse augurer du passage de l'aménagement à la gestion des milieux aquatiques. La préservation des espaces naturels et de ses espèces a fait l'objet de deux directives européennes: la directive « habitats » et la directive " oiseaux » se complétant pour la conservation de la biodiversité. Ces directives ont été à l'origine de décisions françaises de création de $\mathrm{ZICO}$ (zones importantes pour la conservation des oiseaux), et de ZPS (zones de protection spéciale) nécessitant des mesures particulières de gestion ou de protection des populations d'oiseaux sauvages remarquables. D'autres directives ont vu le jour ces dernières années concernant les eaux usées, l'eau potable... A l'échelle nationale, la loi de 1964 a posé les fondements d'un développement coordonné et durable de la ressource en eau, et a fait prévaloir le principe de solidarité entre les usagers. La loi sur l'eau du 3 janvier 1992, qui prolonge la loi de 1964, repose sur les principes suivants :

- l'eau et les milieux aquatiques constituent un patrimoine commun,

- la nécessité d'une gestion intégrée :

- équilibrée entre milieux et usages,

- décentralisée et concertée,

- institutionnelle et pérenne ;

- la mise en œuvre d'outils novateurs de réglementation et de planification.

Cette loi répond à une conception de la ressource appelant davantage :

- de rigueur pour gérer une ressource épuisable ayant une valeur économique,

- d'unité pour concilier usages et valeur patrimoniale des milieux,

- de transparence en suscitant la participation de tous les acteurs à la démarche.

Ce rapport ne constituera pas un état exhaustif de la recherche dans ce domaine, tous les thèmes intéressés par l'eau ne seront pas traités.

Il a pour ambition :

- d'induire une prise de conscience commune de l'état des lieux des hydrosystèmes et des enjeux concernés,

- de dresser un bilan des outils existants, 
- de proposer des remèdes en terme de moyens d'action et de perspectives, en apportant des éclairages sur certains thèmes, et en tentant d'établir des liens entre la recherche et les différentes étapes des processus décisionnels.

\section{CONSTATS ET ALTERNATIVES}

\subsection{L'eau, un patrimoine naturel}

270000 kilomètres de réseau hydrographique, comprenant fleuves, rivières et ruisseaux, courent sur notre territoire, d'importants aquifères y résident aussi. Il y a moins de vingt ans, l'eau était encore parfois considérée comme un élément à maîtriser plus qu'une ressource. Cette évolution est due à un progrès de la connaissance scientifique, à une prise de conscience du public, à des périodes de sécheresse et à une dégradation de la qualité des eaux. La loi sur l'eau de 1992 affirme la valeur patrimoniale de l'eau et déclare d'intérêt général sa protection, sa mise en valeur et son développement dans le respect des équilibres naturels. L'eau est l'élément majeur du fonctionnement des écosystèmes, l'eau et la vie étant indissociables à l'intérieur des systèmes naturels. Aussi allons-nous identifier et caractériser les processus dans lesquels elle est impliquée.

La communication de B. Lachat intitulée « De l'âge de la pierre à l'âge du vert ", dans sa première partie, illustre les propos qui vont suivre en s'attachant à expliquer pourquoi un cours d'eau ne doit plus être considéré comme un « canal évacuateur ».

\subsubsection{Approche multidimensionnelle de l'hydrosystème}

A une vision linéaire du cours d'eau, le concept d'hydrosystème s'est peu à peu substitué, considérant la rivière à l'échelle du bassin versant comme un système complexe, multidimensionnel et composé d'un ensemble d'écosystèmes interagissant entre eux. L'hydrosystème intègre quatre dimensions :

- la dimension longitudinale caractérisée par des flux d'amont en aval, différenciée par des zones (cours supérieur, moyen et inférieur) et une répartition des communautés végétales et animales selon des entités écologiques (zone à truite, zone à ombre, zone à barbeau, zone à brème),

- la dimension transversale illustrée par des flux latéraux intéressant les lits majeurs et mineurs. Axe souvent sacrifié par l'homme pour se prémunir contre les inondations, il est le siège de fonctions vitales (zones boisées rivulaires, corridors fluviaux, annexes fluviales),

- la dimension verticale ciblant les échanges dans le domaine hyporhéique, lieu de contact entre système superficiel et système souterrain, fréquenté entre autres par les invertébrés benthiques,

- la dimension temporelle responsable du remaniement des milieux tant au plan physique (habitats) que biologique (biocénoses) par l'alternance des crues et des étiages et par les phénomènes d'érosion et de sédimentation.

Cette dynamique conduit à identifier, de part et d'autre des cours d'eau, un espace de liberté dans lequel ils sont amenés à évoluer. Elle participe à la diversité biologique en permettant le rajeunissement de certains milieux recolonisés ainsi par des espèces pionnières.

\subsubsection{Les espaces associés aux cours d'eau}

Divers milieux sont associés aux cours d'eau et participent à leur fonctionnement au sein de l'écosystème rivière. Caractéristiques et fonctions définissent chacun d'eux. Ils consti- tuent d'importantes zones de transition entre milieu terrestre et milieu aquatique, offrant une grande variété de biotopes, et où l'exposition à la submersion régit la distribution des communautés végétales et animales. On distingue :

- les milieux associés à l'eau tels les îles, les grèves, les bancs d'alluvions et les vasières. Une durée courte mais suffisante entre deux submersions permettra la colonisation par des espèces fugaces, à la biologie particulière, et dont l'intérêt écologique est élevé,

- les milieux de transition comprenant :

- les berges séparant lit mineur et lit majeur, où la végétation se répartit selon le gradient d'humidité et joue un rôle de stabilisation très important,

- les bras morts et les bras secondaires dont les communautés oscillent, selon le lien à l'écoulement principal, entre celles des eaux courantes et des eaux closes,

- les marais, marécages et tourbières liés à l'engorgement du sol.

- Les milieux terrestres situés dans le lit majeur, tels les landes alluviales, les forêts alluviales, les prairies inondables et les vals cultivés.

Ces zones humides couvrent une faible superficie du territoire national, mais leur importance est primordiale, assurant les écoulements, structurant le paysage et constituant des milieux vivants abritant une grande diversité d'animaux et de végétaux liés entre eux par des relations nutritionnelles.

\subsubsection{Approche fonctionnelle de l'écosystème}

La rivière et ses annexes constituent un système biologique homogène ou écosystème (unité de base des systèmes écologiques complexes). Il est défini par sa structure (biotope et biocénose) et par son fonctionnement (flux d'énergie et de matière). L'analyse de ce dernier s'appuie sur la description de "compartiments " hiérarchisés et de leurs interactions. Il en ressort que les facteurs déterminants du fonctionnement du milieu aquatique ou facteurs de contrôle sont respectivement le bassin versant (écorégion), le régime hydrologique influant la dynamique temporelle de l'hydrosystème, la morphologie et la ripisylve.

Or ces compartiments, cibles des interventions humaines, agissent sur des facteurs clefs du fonctionnement à l'intérieur du milieu aquatique (paramètres physico-chimiques, habitats, ressources alimentaires), ceux-ci étant responsables de la structuration des communautés vivantes. En résumé, des perturbations induites au niveau des facteurs de contrôle provoqueront des modifications en cascade avec répercussion sur la biocénose. Or celle-ci constitue l'édifice biologique (producteurs-consommateurs et décomposeurs) possédant l'aptitude à l'autoépuration et étant ainsi « responsable » de la qualité des eaux. Un édifice biologique altéré réduira la capacité d'assimilation d'un cours d'eau (réduction du nombre d'espèces, développement de groupes tolérants...).

Si régime et qualité des eaux dépendent des aménagements réalisés dans le lit mineur des cours d'eau, ils sont directement liés à l'occupation des sols et aux aménagements réalisés sur le bassin versant. Comme nous l'avons vu précédemment, échanges de matière et d'énergie caractérisent le lien entre habitats-communautés vivantes et qualité des eaux, celle-ci n'étant que la résultante de ces échanges. Ainsi, avoir comme objectifs la satisfaction des usages et l'amélioration de la qualité de l'eau ne peut se concevoir que si le maintien de l'édifice biologique est l'objectif préalable.

\subsubsection{Un système complexe d'échanges entre eaux souterraines et eaux superficielles}

Les eaux souterraines, longtemps considérées comme inépuisables et invulnérables, constituent un patrimoine menacé. En effet, elles ont montré leurs limites quantitatives et qualitatives, la vulnérabilité des aquifères variant selon leurs 
caractéristiques propres. La reconstitution de leur stock s'effectue à partir des eaux d'infiltration en hiver et est ainsi très dépendante de la pluie et de l'hydraulicité. Leur qualité est directement liée à celle des eaux d'infiltration chargées de nitrates, produits phytosanitaires, etc... Ces propos illustrent les relations complexes entre système superficiel et système souterrain. Bien qu'ayant des caractéristiques différentes (temps de renouvellement, accès), ces systèmes ne peuvent plus être traités comme des entités indépendantes.

\subsection{L'eau, une ressource naturelle}

Cette ressource a la caractéristique d'être inégalement répartie dans le temps et dans l'espace. Aussi, les quantités disponibles varient-elles énormément. Cette variabilité explique l'aménagement des cours d'eau pour répondre aux besoins des usagers, mais aussi pour protéger les biens et les personnes des inondations.

B. Mahiou, C. Malusi et P. Spirito, par leur communication concernant le barrage du Puylaurent, illustrent l'aménagement visant à répondre à la demande de certains usages (agriculture, activités touristiques, production d'énergie hydroélectrique) dans une logique de développement économique régional.

\subsection{L'eau de tous les usages}

La consommation d'eau en France en 1990 était distribuée de la façon suivante:

- agriculture : $45 \%$,

- eau potable : $47 \%$,

- industrie : $8 \%$.

L'agriculture utilise des quantités importantes d'eau, sur une durée relativement courte, et prélevées en grande partie dans les cours d'eau en période d'étiage. Son impact est donc prépondérant. L'industrie utilise surtout des eaux de surface et la production d'eau potable s'effectue essentiellement à partir des eaux souterraines.

L'évolution prévisible de la demande en eau est variable selon les usages. Les besoins en eau potable, selon I'OCDE, pourraient passer de $150 \mathrm{l} /$ habitant/j à $280 \mathrm{l} /$ habitant $/ \mathrm{j}$. Ceux de l'industrie décroissent, en partie grâce aux efforts réalisés en matière d'économie d'eau. Il en va de même pour les volumes utilisés par EDF. Par contre, les besoins agricoles ne cessent de croître. La sécheresse de ces dernières années a conduit à un déséquilibre ressources-besoins, et le milieu naturel en a été la victime.

L'homme a, depuis toujours, utilisé le cours d'eau comme source d'énergie, réalisant des ouvrages pour le fonctionnement de ses installations, ceux-ci nécessitant un entretien. Or l'abandon quasi général de ces activités professionnelles et de l'entretien des ouvrages a porté préjudice au fonctionnement du cours d'eau. La transformation de certains d'entre eux en micro-centrales hydro-électriques a rendu difficile la prise en compte des intérêts multiples des cours d'eau.

\subsubsection{L'eau et les activités économiques et de loisirs}

Le cours d'eau et ses espaces associés font partie intégrante du paysage, ils sont des éléments de sa structuration, et donnent caractère et ambiance aux lieux traversés. Aussi, ce paysage est-il souvent à l'origine du développement d'activités de loisirs telles la baignade, la pêche, les sports nautiques, la navigation de plaisance, la randonnée, etc... Ces activités autour de l'eau sont en pleiné expansion, elles traduisent la recherche de qualité de vie. Elles impliquent la mise en place d'aménagements particuliers ayant un impact plus ou moins important sur le milieu et elles ont des exigences en matière de qualité, (baignade, pêche...).

\subsubsection{L'eau, siège de rejets ponctuels et diffus}

Les cours d'eau, sites privilégiés de concentration des activités humaines, constituent l'exutoire naturel des eaux usées tant en rejets ponctuels, épurés ou non (domestiques, industriels, agricoles-élevage) qu'en rejets diffus (domestiques et agricoles). Ces rejets ne sont pas sans incidence sur la qualité des milieux dans lesquels ils se rejettent.

\subsubsection{Milieux aquatiques et extraction de granulats}

L'exploitation des matériaux alluvionnaires dans les lits majeurs et mineurs a connu un tel essor qu'elle n'a pu être sans incidence sur les sites de prélèvements. En conséquence, l'extraction en lit mineur n'est plus autorisée, celle concernant le lit majeur est soumise à certaines conditions qui se retrouvent dans les schémas départementaux des carrières.

\subsubsection{Protection contre les crues}

L'eau, une ressource naturelle, qui a attiré l'homme et ses activités sur les rives, mais qui $a$, par ses excès que l'homme a tendance à oublier, porté préjudice aux personnes et aux biens. L'homme a ainsi cherché à se protéger en tentant de maîtriser cet élément au détriment des besoins de libre divagation des cours d'eau.

\subsection{Un constat}

Les lois de 1964,1976 et 1984 concernant respectivement la lutte contre la pollution et la protection de la nature ont déclenché de gros efforts en vue de reconquérir la qualité des milieux. Une amélioration réelle de la qualité de l'eau due à la dépollution a été constatée, mais on est encore loin des objectifs affichés, les milieux naturels aquatiques étant de plus en plus menacés par le développement économique.

- Des rivières de plus en plus artificielles dues aux modifications lourdes des milieux physiques (barrages,...) ou de la physico-chimie (pollution); le fonctionnement biologique de l'écosystème est alors très perturbé : banalisation des habitats, baisse de la diversité, dégradation de la qualité de l'eau, obstacles aux migrateurs, modification des régimes hydrologiques, impact des vidanges d'ouvrages...

- Des rivières abandonnées, quel que soit leur régime juridique, l'entretien régulier n'étant plus effectué.

- Des rivières pénalisées par des travaux hydrauliques effectués dans le lit mineur (curage, recalibrage, rectification, stabilisation du lit...). Ils ont en général pour objectifs la lutte contre les inondations, l'érosion, l'enfoncement du lit, I'aménagement ou la restauration pour pallier à un manque d'entretien. Leur impact sur le milieu aquatique a été sous-évalué, réduisant la diversité des habitats et donc des communautés. La banalisation des écoulements entraîne une réduction des capacités d'auto-épuration et une moindre résistance aux agressions.

\section{- Des milieux dégradés par l'extraction des granulats}

Ces travaux entraînent souvent des altérations hydrauliques et biologiques graves du milieu, les effets pouvant se répercuter loin des sites d'extraction. Les nappes phréatiques sont rendues plus vulnérables et les zones humides présentes avant ces sites n'existent plus. De plus, le mode de réhabilitation des anciens sites n'est pas sans poser de problèmes.

\section{- Des zones humides en forte régression}

Le rapport d'évaluation concernant les zones humides réalisé en 1994 révèle:

- une très forte régression des milieux d'eau douce telles les landes humides, les prairies humides, les ripisylves et les forêts alluviales, 
- une atteinte moins importante concernant les tourbières, les formations palustres et les eaux courantes lentes,

- une progression en superficie des eaux stagnantes.

Si l'appréciation de l'évolution des milieux et de leur fonctionnement écologique est difficile, sept grands types de problèmes ont été mis en avant :

- l'assèchement par raréfaction des inondations et la baisse de la nappe phréatique,

- les perturbations des échanges hydrauliques entre milieux connexes,

- l'enclavement et le mitage,

- l'eutrophisation et le comblement,

- la pollution chimique,

- la fermeture et/ou la banalisation des milieux par abandon,

- divers problèmes de fonctionnement, tels les prélèvements excessifs, les perturbations hydrauliques.

Le constat concernant les zones humides est dressé dans la communication de G. Barnaud intitulé «Fonctions et rôles des zones humides $"$.

- De nombreuses espèces disparues ou menacées

Les cours d'eau à l'état «originel » (cette notion doit être maniée avec beaucoup de précautions) ont presque entièrement disparu des pays industrialisés. De nombreuses espèces sont d'ores et déjà éteintes ou menacées d'extinction. Une étude réalisée en 1980 pour le Conseil de I'Europe précisait que 103 espèces de poissons d'eau douce étaient menacées, rares, ou vulnérables. Il en va de même pour les mammifères, certains oiseaux et de nombreuses espèces d'amphibiens et de plantes... Les facteurs de dégradation sont multiples et recensés pour la plupart ci-dessus. L'objectif affiché ces dernières décennies consiste à axer tous les efforts sur la lutte contre la pollution, en délaissant la protection des habitats.

- Un paysage maltraité, car pris en compte de manière marginale, ou écarté des dossiers.

On a tenté de répondre à la demande exprimée, aux besoins, et de concilier les usages. L'eau a été maîtrisée dans ce but. Or la situation de «cueillette » où chacun prend ce dont il a besoin a montré ses limites lors des récentes années sèches. Il en est ressorti la notion de priorité ou hiérarchisation des usages en terme de prélèvement sur la ressource en eau. Quant à la pollution, moins de la moitié des objectifs de qualité fixés par les autorités départementales et de bassin sont atteints. Les techniques d'épuration ont amélioré la qualité des rejets, mais le taux de collecte des eaux usées reste insuffisant. De nouvelles formes de pollution apparaissent, et leur caractère diffus les rend plus difficiles à traiter. L'absence de cohérence parfois constatée entre aménagement du territoire et gestion de l'eau, a conduit des activités polluantes à se multiplier dans certaines régions, mettant en péril la ressource en eau.

\subsection{Quelles alternatives?}

La politique d'aménagement des vallées suivie jusqu'à maintenant a généralement consisté à satisfaire des usages déterminés : urbanisation, granulats, électricité, agriculture ; elle a été trop sectorielle et limitée aux aspects hydrauliques et économiques.

La fonction des milieux naturels, qui ne correspond pas à un usage direct mais à une utilité collective, n’a pas été suffisamment reconnue jusqu'à nos jours et leur fragilité prise en compte dans les décisions d'aménagement.

Il est nécessaire de mieux prendre en compte l'interdépendance entre le cours d'eau, sa vallée et son bassin et de tenir compte des effets des aménagements et des modes de gestion de l'espace sur les milieux et les écosystèmes.

Toute perturbation de l'équilibre de ces milieux entraîne une dégradation du niveau et de la qualité de la ressource en eau ainsi que de la diversité et de l'abondance des peuplements du milieu.
Maintenir l'intégrité de leur fonction pour garantir la pérennité de la ressource en eau est un objectif prioritaire.

Il est donc indispensable que les documents de planification soient cohérents avec les exigences des écosystèmes sur les objectifs essentiels en matière de régime hydrologique, de qualité et quantité de l'eau et sur la gestion du territoire des vals en intégrant la préservation des habitats et des milieux naturels.

Le changement de logique pourrait se traduire par le respect des règles suivantes pour les actions envisagées : - adapter les usages aux potentialités réelles du milieu, - améliorer la gestion de l'existant plutôt que d'aménager encore,

- développer une gestion cohérente à l'échelle du bassin versant englobant les opérations d'aménagement ponctuelles, - élargir le choix des techniques en optant pour celles ayant un impact limité sur les milieux, ou celles favorisant une renaturation des sites concernés,

- gérer les eaux et les milieux aquatiques de façon intégrée et concertée, pour assurer un développement durable des usages.

C'est ainsi que B. Lachat, dans sa communication, propose en deuxième partie :

- une philosophie résumée en quelques règles en matière d'aménagement de cours d'eau,

- une méthode d'approche des problèmes d'érosion,

- les techniques du génie végétal, alternatives aux techniques dures pour protéger les berges contre l'érosion.

\section{QUESTIONS AUX AUTEURS}

1) B. Mahiou - SHEMA - Groupe EDF, - C. Malusi - Conseil Général de l'Ardèche et $P$. Spirito - Soc. d'Equipement de la Lozère.

"Le barrage de Puylaurent: un partenariat réussi entre la Lozère, l'Ardèche et EDF ».

- Puy-Laurent et l'aménagement plus ancien de Montpezat, Pont de Veyvière, permettent de soutenir le débit de l'Ardèche de façon complémentaire. Une réflexion at-elle été engagée sur l'optimisation de ces deux ressources pour le soutien de l'Ardèche en intégrant les autres contraintes (et notamment la dérivation du cours de la Loire)?

- A quel horizon cette retenue permettra-t-elle de satisfaire les besoins?

Est-ce l'enveloppe des besoins qui a été prise en compte? Sinon, quel est le risque de défaillance?

\section{Réponse à la question 1}

\section{Malusi :}

L'Ardèche bénéficie depuis cet été de deux ressources importantes situées en amont du bassin:

- Pont-de-Veyrières qui fait sentir ses effets dès les premiers kilomètres de la rivière Ardèche et permet de mobiliser $12 \mathrm{hm}^{3}$ sur les réserves EDF de l'aménagement de Montpezat ;

- Puylaurent situé sur le Chassezac, affluent principal de l'Ardèche qui rejoint cette dernière à Vallon-Pont-d'Arc et apportera à terme $10 \mathrm{hm}^{3}$.

Cette volonté d'équipement de réserves en eau a été assortie de la mise en place d'un tableau de bord regroupant l'ensemble des données hydrologiques du bassin de l'Ardèche: état des réserves, débits de la rivière en des points caractéristiques, débits de soutien... Ce dernier est consultable sur un réseau informatique par les responsables de sa gestion et les Services de contrôle. La conception de cet ensemble a bénéficié des concours techniques d'EDF et de la DIREN Rhône-Alpes. Le point de convergence des 
lachures des deux réservoirs étant situé juste en amont des gorges, les deux barrages se complètent fort bien et fonctionnent indépendamment lorsque les réserves totales sont constituées en début de saison estivale.

L'optimisation que vous évoquez interviendra en situation de défaillance de constitution des stocks d'eau et ce d'autant plus que cette défaillance sera profonde. Pour l'aider à fixer les objectifs particuliers en situation critique, le Département est conseillé par un Comité de Gestion qui regroupe les partenaires et les Services de l'Etat. Un outil d'aide à la décision est en cours d'élaboration. Toutes les données relatives aux débits estivaux dans le bassin de l'Ardèche. qu'elles soient gérées par le Ministère de l'Environnement ou EDF, sont collectées grâce à un système de boîtes à lettres piloté par un serveur qui, après traitement, les restituera sous forme de graphiques et de bases de données.

\section{B. Mahiou :}

En complément, pour répondre totalement a votre question. vous avez évoqué, en tant qu'homme de la Loire, la dérivation opérée par l'aménagement hydroélectrique EDF de Montpezat. Je vous répondrai en signalant tout simplement que la réalisation de l'opération de Pont-de-Veyrières n'a apporté aucune modification dans la gestion des réserves en eau du haut-bassin de la Loire en terme de volumes d'eau dérivés. Ces derniers sont inchangés. L'accord signé avec EDF a juste prévu qu'une partie des volumes stockés au début de la période estivale seront libérés en été pour le soutien d'étiage de l'Ardèche au lieu d'être turbinés en hiver Pour le seul bénéfice énergétique.

En cas de défaillance hydrologique, EDF respectera en premier lieu son cahier des charges de concession hydroélectrique, ce qui l'amènera à soutenir l'étiage de la Loire en période estivale (ce qu'elle a déjà fait par le passé) en même temps qu'elle assurera le Soutien de l'Ardèche à hauteur des volumes d'eau disponibles par l'intermédiaire du barrage de Pont-de-Veyrières.

\section{Réponse à la question 2}

\section{B. Mahiou :}

Les volumes de soutien d'étiage et d'irrigation sont prévus en progression afin de prendre en compte l'accroissement des besoins en eau pendant cette période tels qu'ils ont été définis dans le cadre d'une étude réalisée par la DIREN Rhône-Alpes :

- $1996: 7,5 \mathrm{hm}^{3}$

- de 1997 à $2004: 8.5 \mathrm{hm}^{3}$

- de 2055 à 2009: $9 \mathrm{hm}^{3}$.

- à partir de 2010: $10,1 \mathrm{hm}^{3}$.

Bien entendu, le stockage de ces volumes dans la retenue, dont la capacité est de $12 \mathrm{hm}^{3}$, est assorti d'une défaillance, le taux de défaillance pour le remplissage total variant de 5 années sur 100 à 12 années sur 100 suivant les volumes d'eau à mobiliser. Dans cette situation pénalisante, un comité de gestion de l'eau placé sous la présidence du Préfet coordonnateur de bassin et averti suffisamment à l'avance par l'exploitant EDF, rendra les arbitrages nécessaires à la répartition des volumes d'eau disponibles.

Vous noterez ici que l'intérêt de cette opération à buts multiples pour les collectivités locales est de pouvoir bénéficier au moment voulu des volumes d'eau requis. Lorsque l'eau n'est pas utilisée pour le soutien d'étiage ou l'irrigation, EDF peut la turbiner en hiver au moment où elle en a besoin. Le montage financier a d'ailleurs intégré cette progression de la demande en eau des Collectivités. Leur participation financière a été ainsi chiffrée en manque à gagner pour EDF qui sait parfaitement estimer la valeur d'l $\mathrm{m}^{3}$ d'eau transformé en $\mathrm{kWh}$. Une clause conventionnelle a également prévu que des modifications puissent être apportées au calendrier des besoins en eau à un prix convenu d'avance.

\section{Malussi :}

Pour terminer, je dirai que le premier soutien d'étiage a eu lieu cet été faisant suite à la première mise en eau du barrage. Il s'est parfaitement déroulé.

2) B. Lachat - Biotec.

"De l'âge de la pierre à l'âge du vert".

- En quoi le génie végétal est-il une technique alternative ? Quels sont ses domaines d'application et ses buts ?

- Quelles sont les questions à se poser devant un problème d'érosion de berge ?

- Pouvez-vous résumer quels sont vos principes en matière d'aménagement ?

\section{Réponse à la question 1}

Le génie végétal utilise essentiellement des plantes pour résoudre les problèmes de l'ingénieur en matière d'érosion et de glissement de sols. Les végétaux constituent des " matériaux de construction " vivants et ne sont, bien évidemment, pas utilisés comme cosmétiques!

Les avantages, bien connus maintenant, peuvent se résumer à :

- effet de stabilisation dynamiquement croissant ;

- souplesse des ouvrages et résistance (ancrage dans la berge et non appui contre elle; tensions d'arrachement ou de glissement reprises en traction):

- actions hydromécaniques de la végétation sur et dans le sol : interception, absorption et transpiration d'eau, augmentation de la cohésion du sol, effet de cintrage des racines, etc...; ;

- impacts de chantiers minimaux :

- amélioration de l'autoépuration du cours d'eau :

- augmentation de la biodiversité et de la valeur du milieu (support-abri-nourriture) ;

- embellissement du paysage et intégration parfaite des ouvrages dans le site aussi bien en milieu rural qu'urbain; - gains financiers généralement entre $40 \%$ et $90 \%$ sur les méthodes classiques :

- si travaux de mêmes coûts que le génie civil: résultats écologiques et paysagers meilleurs; ouvrages vivants donc pérennes:

- fourniture des matériaux souvent sur place: transports simplifiés, piste de chantier inutile :

- matériaux de construction bon marché et bilan écologique global avantageux :

- exploitation possible des produits issus de la croissance des ouvrages (permet un revenu ou fournit des matériaux à bon compte pour d'autres ouvrages):

- etc...

Le génie végétal constitue donc une alternative très sérieuse aux techniques classiques du génie civil.

Les domaines d'application sont les pentes et talus (chemins de fer, routes, zones naturelles, etc...), les berges des cours d'eau et des canaux (navigués ou non), ainsi que les autres zones nécessitant le maintien des sols et la lutte contre l'érosion ou les glissements.

Le but essentiel du génie végétal, outre ses fonctions techniques, est d'assurer le maintien ou l'amélioration des fonctions naturelles (composantes écologiques) du site où il est employé.

\section{Réponse à la question 2}

L'analyse du milieu récepteur de l'ouvrage est indispensable, ainsi que la parfaite compréhension des phénomènes érosifs et des contraintes. Parmi les facteurs importants à noter, on peut citer : 
a) eau :

- débits et niveaux de crue ;

- force tractrice ;

- submersion ;

- périodicitélfréquenceldurée des crues resp. des étiages :

- batillage;

- percolation:

- etc...

b) sol:

- fertilité :

- constitution:

- stabilité, cohésion:

- pente.

- etc...

c) climat :

- exposition :

- lumière;

- altitude;

- durée du gel:

- enneigement;

- etc...

d) site :

- végétation existante :

- occupation des sols riverains :

- obstacle, embâcle.

En fait, les questions à se poser sont quasiment les mêmes que pour les techniques des domaines de l'ingénierie classique. Cependant, il faut tenir compte du fait que l'on travaille avec des végétaux et que la qualité des sols et le climat ont une importance primordiale.

L'expérience compte beaucoup dans l'appréhension de ces facteurs car les végétaux ne se mettent, malheureusement. pas en équation. Il est très difficile de rendre reproductible une situation donnée. Cela montre également qu'il n'est pas souhaitable, comme on le faisait couramment jusqu'ici, de simplifier et de banaliser l'ensemble de ces facteurs.

\section{Réponse à la question 3}

Comme l'objet de mon intervention ciblait principalement les cours d'eau, je vais cadrer ma réponse par rapport à ces éléments qui sont les plus touchés par rapport aux divers domaines du génie végétal.

Partant du constat qu'en cours d'eau de plaine, les blocs de rocher ne "poussent "pas spontanément sur les berges mais que la végétation, elle, en est souvent capable, il faut logiquement privilégier les techniques du génie végétal. En fait, si l'on se place au niveau du cours d'eau et de tout ce qui y vit (y compris l'homme qui l'utilise) le traitement des érosions ou les aménagements doivent répondre à la hiérarchie logique suivante:

1. Faut-il réellement intervenir sur le cours d'eau?

Cela dépendra des enjeux, des impacts prévisibles, du paysage, du résultat écologique, etc...

2. Evaluer si les techniques végétales peuvent satisfaire à la résolution des problèmes.

Dans ce cas, favoriser au mieux la biodiversité, pour autant que cela ne nuise pas à l'efficacité de l'ouvrage.

3. En cas d'impossibilité ci-dessus, établir si des techniques combinées peuvent pallier aux problèmes.

4. Appliquer, seulement à ce stade, une technique classique de génie civil, pour autant qu'elle s'avère raisonnable et proportionnée.

D'une manière plus générale, en relation avec le génie végétal face à un problème d'érosion donné, il est plus favorable d'agir de manière à donner un "coup de pouce " à la Nature pour qu'elle se développe et se "débrouille "toute seule. Avec l'observation et la compréhension des phénomènes érosifs d'une part, avec une bonne connaissance du milieu récepteur des aménagements d'autre part, on va concevoir et réaliser un minimum d'interventions pour traiter davantage la cause de l'érosion plutôt que de " colmater " à grands frais l'érosion elle-même. 


\section{III — LES ENJEUX}

S'il est banal de parler de la nécessité vitale de l'eau, la conscience des enjeux qu'elle représente n'est pas aussi évidente. Ceux-ci sont multiples et ils constituent une étape d'analyse importante dans le processus décisionnel préalable à l'aménagement d'un cours d'eau et de ses espaces. La perspective de contraintes économiques et financières pour les années à venir appelle une évaluation claire des enjeux liés à la préservation de l'environnement dans ses dimensions économiques, sociales et écologiques. Celles-ci sont définies par les objectifs des politiques publiques de l'environnement soit : préserver la diversité biologique, améliorer la qualité de la vie, assurer la sécurité des populations contre les risques, permettre un développement durable des ressources et transmettre aux générations futures un patrimoine.

\subsection{Enjeux économiques}

Actuellement, l'évaluation des projets d'investissement est réalisée par le calcul économique. Celui-ci doit être manié avec prudence du fait du choix d'un taux d'actualisation qui influe sur l'évaluation de la rentabilité totale d'un projet, ainsi que par l'effet « intergénérationnel » qui conduit nécessairement à arbitrer entre le bien-être de deux générations.

C'est ainsi que le coût des dommages peut être supporté par une génération qui n'a pas fait le choix de l'aménagement. Aujourd'hui, l'évaluation de l'impact des projets d'investissement sur les actifs naturels ne prend généralement pas en compte les effets d'irréversibilité qui peuvent entraver le renouvellement de ces ressources ainsi que leur pérennité.

Ce constat conduit à penser que le seul calcul économique ne peut suffire à évaluer un projet réalisé aujourd'hui ayant des incidences sur plusieurs générations. L'économie de l'environnement doit, au-delà d'une analyse financière, évaluer les effets induits par les choix d'investissement sur l'environnement et sur les acteurs économiques impliqués. Il s'agit de mesurer l'impact des choix actuels sur la pérennité du patrimoine eau de demain.

\section{I.I Les conclusions de l'approche économique du SDAGE Loire-Bretagne}

Le but n'est pas de traiter la théorie de l'économie de l'environnement, mais d'exposer quelques enseignements tirés de l'approche économique du SDAGE du bassin Loire-Bretagne.

Les objectifs de la démarche consistaient à :

- mettre en évidence les investissements induits par les politiques de préservation et de pérennisation de la ressource en eau, et les bénéfices engendrés,

- affecter une valeur aux divers usages en évaluant leur coût de dégradation et de leur perte,

- mettre au point une méthodologie d'évaluation économique.

Sept thèmes ont été étudiés. Chacun a fait l'objet d'une analyse coûts-bénéfices. Deux scénarios ont été testés sur chaque thème :

- un scénario " tendanciel " reflétant les pratiques actuelles et leur poursuite,

- un scénario de pérennisation de la ressource incluant mesures curatives et préventives.

Ces scénarios ont été testés sur des zones échantillons. Le faible nombre de données locales et la difficulté de prendre en compte des effets à long terme doivent inciter à la prudence quant à l'utilisation des chiffres avancés dont il ne sera pas question ici puisqu'intéressant le bassin Loire-Bre- tagne seulement. Toutefois, ce protocole a conduit aux constats suivants :

- le chiffrage des dépenses à réaliser pour préserver la ressource qualitativement et quantitativement a fait ressortir la prédominance des investissements à réaliser dans le domaine agricole,

- il est difficile d'évaluer les bénéfices par thème compte tenu du décalage entre les investissements à réaliser aujourd'hui et les bénéfices environnementaux constatés à moyen ou long terme (retour de l'investissement décalé),

- la détérioration de la qualité peut induire une réduction des chiffres d'affaires et une diminution des emplois dans certaines industries nécessitant une eau de qualité (conchyliculture, tourisme...),

- certaines préconisations peuvent entraîner des surcoûts pour certaines branches industrielles, ceux-ci étant répercutés sur plusieurs acteurs économiques dont les consommateurs. Ces mesures évitent le coût de dommages écologiques plus importants,

- l'étude relative aux inondations montre qu'une croissance de l'urbanisation augmente les dommages d'autant plus que la zone était peu urbanisée auparavant. Il convient donc de mettre en œuvre une politique d'aménagement du territoire intégrant la gestion du risque. Le maintien de zones d'expansion de crues permet de réduire les dommages de manière importante,

- une répartition judicieuse des sols cultivés est une politique d'aménagement du territoire économiquement rentable, - l'analyse des scénarios curatif et préventif testés sur la qualité nitrate des eaux conduit à penser que la protection préventive est plus onéreuse à court et moyen terme que le curatif (sur 15 ans), le préventif étant d'autant plus cher que l'eau est plus polluée. Par contre le coût baisse à long terme et permet la pérennisation multi-usages. La solution curative est moins coûteuse sur 15 ans, mais elle est à renouveler à ce terme. Son coût à long terme est donc au moins constant, voire croissant. De plus, cette solution ne protège que vis-à-vis d'un seul usage (eau potable...).

L'évaluation de la rentabilité de ces solutions dépend de l'échelle de temps dans laquelle on se place, et de l'objectif de protection mono-usage ou multi-usage que l'on se fixe. La logique de développement durable s'inscrit dans une gestion multi-usage tournée vers les solutions préventives.

\section{I.2 Intérêt économique des zones humides}

L'intérêt économique de ces « infrastructures » naturelles réside :

- dans leur production végétale potentiellement exploitable (bois, roseaux...),

- dans les activités économiques dont elles permettent le développement (chasse, pêche, promenade, loisirs divers...),

- dans leur hospitalité vis-à-vis des espèces animales. En effet, deux-tiers des poissons consommés dans le monde passent une étape de leur cycle en ces lieux.

Leur reconnaître une valeur et constater qu'elles régressent n'est pas suffisant pour faire infléchir cette tendance. Si leur transformation s'est justifiée à une époque par l'amélioration des conditions sanitaires et l'évolution de l'agriculture, des études montrent qu'elles n'apportent pas de gain social global immédiat. Les choix publics, quand il existe des alternatives d'aménagement, sont le plus souvent éclairés seulement par le calcul économique.

D'autres impératifs sont à prendre en compte, mais l'évaluation économique des coûts et avantages reste un élément de décision important... Aussi, la prise en compte des zones humides passe-t-elle par une identification de la valeur des services non marchands qu'elles génèrent, mais aussi par une politique commune des divers départements ministériels vis-à-vis de ces espaces. 
Les zones humides remplissent des fonctions que nous verrons ci-après, que l'on peut monétariser, et leur transformation n'est pas toujours économiquement rentable. En effet, de nombreux marais drainés et remembrés afin d'être cultivés sont retournés en friche à cause de leur faible rendement, et abandonnés par leur propriétaire, celui-ci ne trouvant pas acheteur.

\section{- 3.2. Enjeux sociologiques}

La période actuelle est caractérisée par une prise en charge partagée de l'environnement, entre les pouvoirs publics et la société (associations, consommateurs,...). Ainsi la demande sociale jouera probablement un rôle important dans l'arbitrage entre économie et écologie, et aidera à la définition de priorités.

La difficulté réside dans le fait que cette demande sociale n'est pas cernée, car mal connue et ambiguë. Il existe peu d'outils disponibles pour évaluer cette demande, en dehors des sondages d'opinion. Or, on ne peut réduire ces enjeux à l'évolution des résultats des sondages et aux changements des comportements individuels. Les comportements de groupes et leur évolution sont tout aussi importants puisqu'ils sont, dans le domaine qui nous intéresse, porteurs de concertation, de conflits, de décisions...

Au-delà de ces réserves, il est possible d'affirmer que de nouvelles exigences se font sentir:

- La demande en eau est considérée comme un impératif, et un défaut de disponibilité ne doit pas constituer une entrave au développement économique lié à son usage. Mais on déplore en même temps que le milieu naturel en paie le prix. A ressource fluctuante, correspond une grande variabilité des besoins en eau. Si la demande en eau potable est actuellement à peu près stabilisée, l'agriculture a des besoins croissants durant les périodes d'étiage.

- L'eau est souhaitée de qualité dans un but d'hygiène publique. Si la qualité sanitaire des eaux de baignade et de l'eau potable s'est améliorée, il n'en est pas de même pour les eaux conchylicoles.

-. Le consommateur est de plus en plus exigeant vis-à-vis de la fiabilité de l'alimentation en eau potable, de la transparence des coûts, mais aussi de l'information concernant le produit « eau » (origine, qualité...).

- Les populations vivant dans les zones inondables attendent de la collectivité qu'elle assure leur sécurité face aux inondations.

- Ces dernières années, une tendance nouvelle est apparue : la redécouverte de la navigation pour le transport et surtout le tourisme. Le projet de développement du transport fluvial s'inscrit dans le cadre de l'aménagement du territoire à l'échelle nationale et européenne. Quant au tourisme fluvial, sous la pression d'une demande importante, il se développe et fait l'objet de nombreux projets.

- L'amélioration du cadre de vie constitue une exigence partagée par tous. Les villes sont la première cible, les habitants attendant leur réconciliation avec leurs eaux, et l'offre de loisirs autour du milieu aquatique.

- Les enquêtes montrent que cette génération est très attachée à léguer aux générations futures un patrimoine eau et environnement de qualité.

Les diverses exigences citées ci-dessus émanent d'individus représentant ce qu'on a l'habitude d'appeler l'opinion publique, et de groupes d'individus représentant un usage ou la défense d'un thème (associations). Or ces publics ont des attentes différentes vis-à-vis de cette eau qui est leur " ciment ", on peut alors se poser la question suivante : «Parlent-ils tous de la même eau »?

\subsection{Enjeux écologiques}

Ces trente dernières années, l'amélioration de la qualité de l'eau a concentré beaucoup d'efforts. Or la nature de la pollution évoluant, la pression des usages en terme de prélèvement croissant, et l'artificialisation des milieux augmentant, les résultats obtenus n'ont pas été à la hauteur de ce que l'on aurait pu escompter. Les années 90 connaissent une amplification des exigences écologiques, aboutissement d'une recherche pluridisciplinaire, et la fin d'une approche sectorielle de l'eau. Cette transition s'appuie sur de nombreux engagements internationaux et européens, et notamment sur les directives européennes concernant la qualité des eaux et la préservation de la nature (directive sur les eaux usées urbaines de 1992, directive nitrates de 1991, directive habitats de $1992 \ldots .$.$) .$

\subsection{Les fonctions des milieux aquatiques}

On désigne par «fonctions », les propriétés de "l'écosystème rivière " découlant de son fonctionnement, et ayant une influence positive sur la plupart des besoins de l'homme ou sur les activités s'y développant.

On reconnaît, à ces lieux de transit d'une ressource mobilisable, les propriétés suivantes :

- La régulation hydraulique. Elle se manifeste par l'expansion des hautes eaux dans les zones inondables les stockant pour les restituer progressivement, par l'effet de la végétation dispersant les lignes de courant et diminuant ainsi puissance et force érosive de l'eau, mais aussi par le renforcement des débits d'étiage. De nombreux milieux assument cette fonction : forêt alluviale, landes alluviales, vals cultivés, prairies inondables, bras morts, marais, berges...

- La régulation hydrique. Elle concerne les échanges d'eau air-nappe-rivière par les processus d'infiltration, de restitution et d'évaporation, la végétation participant à ces mécanismes. Tous les espaces permettant l'infiltration de l'eau dans le sol et sa restitution participent à cette fonction (forêts alluviales, bras morts et secondaires, berges, landes et prairies inondables).

- L'autoépuration. Elle comprend une épuration physique et une épuration biologique. L'épuration physique consiste en un piégeage d'éléments par sédimentation et s'exerce essentiellement sur les eaux de ruissellement en provenance du lit majeur ainsi que sur l'eau de la rivière en période de crues. L'épuration biologique concerne les éléments nutritifs : nitrates, phosphates, matières organiques et autres composés tels les métaux, les produits phytosanitaires... Elle est le siège de transformations telles la minéralisation, l'assimilation par les végétaux etc... Cette fonction est directement liée à la présence et à la nature de la végétation mais aussi aux communautés animales présentes. La végétation des forêts alluviales, les berges végétalisées, les bras morts et secondaires contribuent largement à cette fonction.

- La biodiversité. L'écosystème rivière comprend des milieux variés s'étendant du milieu aquatique au milieu terrestre. Il définit ainsi des biotopes organisés selon un gradient d'humidité pouvant varier dans l'espace et dans le temps, d'où la coexistence d'espèces à cycle court et d'espèces stables. Ces milieux sont sources d'abris, de nourriture et des lieux de reproduction pour de nombreuses espèces.

- La valeur paysagère. Le fond de vallée constitue une unité paysagère à valeur élevée tant par la diversité des milieux qu'il propose que par l'organisation de ces derniers autour du cours d'eau.

Comme nous l'avons vu au cours de ce chapitre, la ripisylve (ou formations végétales arborescentes se développant le long des cours d'eau à l'interface milieu aquatique et ter- 
restre) a un rôle majeur. Elle présente une biodiversité végétale importante et une forte productivité grâce à la dynamique fluviale. On peut résumer ainsi les fonctions de la ripisylve :

- Elle intervient sur la morphologie des cours d'eau comme élément de fixation et de stabilisation des berges.

- Elle favorise, à l'échelle du lit majeur, la diversité des habitats piscicoles et crée, par les embâcles, une diversification des habitats pour la faune.

- Elle procure ombrage et limite l'élévation de la température de l'eau.

- Elle limite l'érosion des sols.

- Elle intervient dans la chaîne trophique.

- Elle constitue une zone tampon protectrice pour les cours d'eau (pouvoir épurateur vis-à-vis des nitrates).

- Elle est une voie de migration et un habitat pour de nombreuses espèces (oiseaux et mammifères notamment).

Hydrosystème et ripisylve forment un ensemble complexe, indissociable et essentiel au fonctionnement du système.

G. Pinay, dans sa communication intitulée "Recyclage de l'azote dans les zones riveraines des cours d'eau ", souligne l'importance de cette interface entre milieu aquatique et milieu terrestre, et rappelle les processus intervenant lors du recyclage de l'azote, ainsi que les facteurs de régulation.

\subsubsection{Variabilité et diversité}

La variabilité est une caractéristique importante des cours d'eau. On distingue :

- la variabilité spatiale s'exprimant par une évolution des caractéristiques biologiques, chimiques et physiques du cours d'eau d'amont en aval, mais aussi transversalement,

- la variabilité temporelle annuelle et interannuelle illustrée par une succession de crues, de hautes eaux, d'étiages, d'années sèches et d'années humides.

Cette double variabilité est source de diversité des habitats, des communautés vivantes et des fonctions exercées par les milieux concernés. Elle contribue à la richesse biologique de ces espaces alluviaux qui offrent un couloir de migration aux diverses espèces et qui, de par leurs remaniements fréquents, des vitesses de courant variées, des interrelations entre différents milieux, rendent essentielle leur existence pour une persistance des nappes alluviales satisfaisantes en qualité et en quantité. En conséquence, les réflexions d'aménagement du territoire et plus particulièrement celles concernant les zones inondables devront intégrer la diversité biologique.

Cette richesse biologique est largement illustrée par la communication de G. Verniers intitulée «Le cours d'eau : rôles écologiques et paysagers ». Y sont décrits les composantes de l'écosystème : biotopes et biocénoses, leurs relations, ainsi que les éléments d'appréciation du paysage, d'où des recommandations en matière de gestion.

\subsubsection{Impact des activités humaines sur l'écologie des milieux}

L'impact des activités humaines peut se faire ressentir de différentes manières sur les milieux :

- l'enfoncement du lit, mal très répandu résultant d'une rupture d'équilibre entre énergie disponible et dissipation de celle-ci. Les interventions de I'homme peuvent amplifier l'action de l'eau sur le fond par l'endiguement des berges, le sous-dimensionnement des ponts, le blocage et l'extraction du débit solide, tout ceci entraînant un surcroît d'énergie à dissiper.

Les conséquences sont importantes sur le plan écologique et économique : enfoncement des nappes phréatiques, abais- sement de la ligne d'eau et déstabilisation des fonds entrâ̂nant l'assèchement de milieux connexes, mais aussi le déchaussement d'ouvrages, l'assèchement des puits de captages et l'aggravation des crues en aval :

- la dégradation de la qualité de l'eau a une incidence directe sur les communautés végétales et animales ainsi que sur les habitats (colmatage de frayères...).

- la dégradation quantitative par diminution des débits des cours d'eau due aux prélèvements et dérivations a un impact important sur :

- l'écologie du lit mineur (diminution de la vitesse, augmentation de la température favorisant l'eutrophisation),

- les milieux riverains: assèchement des zones humides, enfoncement des nappes, appauvrissement des biocénoses ;

- la maîtrise des inondations par endiguements, ouvrages régulateurs a conduit sur le plan écologique à une banalisation des biocénoses. En effet, la crue sélectionne les espèces végétales moins communes, elle apporte éléments nutritifs, semences, elle recharge la nappe et tisse un lien entre cours d'eau et milieux humides offrant à la faune: lieu de reproduction, cache et nourriture,

- l'agression vis-à-vis des nappes se traduit par leur pollution, l'abaissement des niveaux piézométriques. La circulation d'eau entre le bassin versant, les cours d'eau et les nappes rend ces dernières indispensables sur le plan écologique notamment pour l'alimentation des cours d'eau en période sèche et pour les besoins en eau de la forêt alluviale, - l'altération de la dynamique fluviale limite la diversité des habitats liés à la submersion et sa durée, et ainsi, de ses communautés. Elle entraîne un vieillissement des milieux riverains,

- la déconnexion des milieux entre eux : l'action de l'homme conduit souvent, par méconnaissance, à traiter de manière isolée milieu aquatique-milieu de transition (berge) et milieu riverain. Or le cycle d'un animal l'amène à fréquenter divers milieux en fonction d'une recherche de nourriture, de frai, de cache, le continuum étant assuré par l'eau et la végétation. Cette rupture entre milieux a une incidence forte sur la faune et contribue à la régression des zones humides.

Les activités humaines pourront ainsi altérer les fonctions de ces espaces. Les endiguements, les ouvrages régulateurs de débit, les dispositifs stabilisant le lit mineur modifieront la capacité de régulation hydraulique de ces milieux. La régulation hydrique sera altérée par une imperméabilisation de substrat, une limitation des surfaces de contact (endiguements, enrochements etc...). La fonction d'épuration peut être amoindrie par des modifications du couvert végétal et de l'occupation des sols. La biodiversité sera altérée par une artificialisation du régime hydraulique et des interfaces (berges, îles...). La fonction paysagère se verra modifiée par des travaux de restauration, des ouvrages régulateurs, des aménagements divers ainsi que par certaines activités urbaines, agricoles et sylvicoles mal maîtrisées.

L'espace fluvial est le siège de nombreux enjeux. Sa gestion nécessitera la prise en compte de l'ensemble des éléments le composant (caractéristiques, fonctions, usages...). Pour ce faire, une bonne connaissance des milieux et de leurs fonctions est nécessaire, l'étude des contraintes et des usages s'y ajoutant pour permettre la détermination des objectifs de gestion.

Les actions de I'homme modifient les milieux et perturbent le fonctionnement des écosystèmes. Il est donc indispensable d'analyser les conséquences de ces actions caractérisées par des degrés d'irréversibilité. Il semble nécessaire d'intégrer la notion de réversibilité des altérations imposées au milieu dans l'analyse des enjeux préalable aux décisions d'aménagement. 


\section{QUESTIONS AUX AUTEURS}

1) G. Pinay - Université de Rennes.

"Rôle des berges dans la rétention de l'azote».

La communication présentée fait largement référence aux conditions rencontrées dans la vallée de la Garonne. - Quelle est la reproductibilité des mécanismes décrits sur de petits cours d'eau très riches en nitrates et souvent sollicités pour l'AEP, soit par le biais de retenues, soit au fil de l'eau?

Le rôle de la ripisylve, du point de vue de la lutte contre la pollution par les nitrates (dénitrification et consommation) semble être mis en évidence là où la rivière est alimentée par une nappe ou alimente ellemême une nappe alluviale.

- En matière de programme d'action, cela revient-il à dire que l'outil « ripisylve » est à réserver à ce seul type de rivière?

- A-t-on des éléments sur la relation entre les dimensions nature de la ripisylve (espèces) et l'efficacité en matière de dénitrification?

- Sait-on finalement bâtir une ripisylve en fonction d'un type de rivière et d'un objectif de qualité ?

\section{Réponse aux questions}

1) Les ripisylves sont des habitats naturels qui, potentiellement, peuvent retenir les pollutions azotées diffuses provenant des bassins versants : elles sont plus efficaces vis-à-vis des apports de subsurface le long des petits cours d'eau. elles sont plus efficaces le long des grands cours d'eau pour la rétention des matières en suspension et des nutriments adsorbés qui proviennent des eaux de surface de débordement durant les épisodes de crues.

2) Les capacités intrinsèques des ripisylves sont inhérentes aux conditions géomorphologiques locales. De même que chaque rectification de cours d'eau a nécessité une étude de terrain précise, la détermination des capacités tampons nécessite aussi une étude de terrain.

3) Nous venons d'organiser avec 3 collègues anglais, du 30 août au 3 septembre 1996, en Grande-Bretagne, un colloque qui avait pour titre "Buffer zones: their processes and potential in water protection „qui a réuni 140 spécialistes de 20 pays différents sur les zones tampons. Un livre sur l'état des connaissances sur ce sujet devrait sortir en librairie au tout début de l'année prochaine. 


\section{IV $\square$ OUTILS DE DIAGNOSTIC ET DE GESTION}

Ces dernières années, la recherche a contribué à une meilleure compréhension du fonctionnement des systèmes, par discipline : I'hydrologie par les modèles QdF (débitdurée-fréquence), 1'hydraulique grâce à l'évolution des modèles numériques de simulation des écoulements, la biologie grâce à la modélisation des paramètres physiques des habitats aquatiques, la géomorphologie par la mise en évidence des phénomènes de dynamique fluviale, la chimie en améliorant la compréhension des mécanismes de transfert des polluants, etc... Le passage d'une approche sectorisée de la ressource en eau à une gestion du cours d'eau et de ses différents usages nécessite une approche globale et intégrée faisant appel à la mesure, à l'évaluation d'impact, à la simulation prévisionnelle. Les outils devront s'adapter à de nombreuses exigences actuelles incitant aussi bien à l'analyse fine qu'à l'approche globale.

\section{- 4.1. Outils de diagnostic}

Ils reposent sur des données concernant le milieu physique et l'impact des activités économiques sur ce milieu. Elles sont collectées, en majorité, par le biais de réseaux de mesures intégrant divers paramètres, tels la climatologie, I'hydrologie, I'hydrogéologie, la qualité des eaux, les prélèvements et les rejets. L'ancienneté du recueil de ces données est variable selon les paramètres.

Concernant l'impact des activités économiques, on note que les prélèvements en nappe et rivière sont bien connus pour les usages domestiques et industriels, moins cernés pour les usages agricoles. Les rejets ponctuels sont identifiées et suivis, et la gamme des mesures réalisées s'élargit progressivement. Les nappes font l'objet d'une bonne connaissance en matière de points de prélèvements et de débits pour l'usage AEP. Pour l'usage irrigation, la situation est moins satisfaisante. La surveillance de leur niveau fait l'objet d'un réseau se mettant en place. C'est sur les aspects qualitatifs que nous enregistrons un retard important, se comblant peu à peu par l'installation de réseaux de mesure. Quant à l'amélioration de la gestion qualitative des retenues, elles nécessitent une bonne connaissance des processus d'échanges eau-sédiment ; c'est le propos de V. Ruban dans sa communication intitulée «Prise en compte du phosphore des sédiments pour une meilleure gestion des écosystèmes aquatiques - Application à la retenue hydroélectrique de Bort-les-Orgues (Massif Central) ».

$\mathrm{Ph}$. Degardin, $\mathrm{Ph}$. Lohest et L. Pereira-Ramos, dans leur communication intitulée «Les mesures sur le milieu et les usages de l'eau, révélatrices des outils de gestion ", exposent ces outils de connaissance du milieu et des usages dans la première partie de leur propos.

L'évaluation de la qualité des milieux aquatiques doit être l'aboutissement d'approches physico-chimiques et biologiques. Recherches fondamentale et appliquée doivent contribuer à la mise au point d'indices biologiques, rendue difficile par peu de connaissances sur les peuplements de référence et la typologie des systèmes, peu de données en biologie et en écologie, une grande variabilité des écosystèmes. L'analyse physico-chimique trouvera une complémentarité dans l'analyse biologique, les organismes vivants étant intégrateurs d'événements (pollutions, modifications), de caractéristiques du milieu, de la fonctionnalité de l'écosystème.

C'est ainsi que la pertinence de la mesure physico-chimique, l'utilisation des bio-indicateurs et l'harmonisation des méthodes, la lisibilité des résultats et leur contribution au diagnostic de cause et à la prévision des évolutions, alimentent les réflexions actuelles concernant la mesure. Sa contribution à l'évaluation de l'impact des activités humaines sur les milieux aquatiques permettra de cerner les degrés de réversibilité des altérations dont ils sont l'objet.

\subsection{Outils de gestion}

En matière de modélisation il ressort deux types de demandes : - une demande locale, pour la gestion d'un ouvrage par exemple,

- une demande globale à l'échelle du bassin versant ou de l'hydrosystème dans un but de gestion intégrée, proposant le test de scénarios, la gestion prévisionnelle, la hiérarchisation et la planification d'actions.

Ce chapitre, convergeant avec la question I du thème II, sera abordé au travers de deux communications illustrant les propos préalables.

J. Eon et G. Merle, dans leur communication intitulée «Contribution de la méthode des micro-habitats au choix du débit réservé ", proposent un outil d'aide à la décision pour la gestion d'ouvrages. Le renouvellement de titres administratifs de nombreuses chutes hydroélectriques est en cours, et nécessite une étude d'impact comprenant le choix d'un débit réservé. L'incidence de ce dernier sur l'écologie des milieux est difficile à appréhender. La méthode proposée par EDF consiste à décrire et simuler le milieu aquatique concerné en fonction du débit, et à évaluer une capacité d'habitat potentiel. Elle comprend le couplage d'un modèle biologique traduisant les relations entre présence d'une espèce piscicole et paramètres caractéristiques de son habitat, et d'un modèle hydraulique calé sur des mesures à plusieurs régimes de débits. Elle permet une comparaison entre l'état actuel de l'habitat et des hypothèses d'évolution de celui-ci en fonction des débits testés. Les effets sur le milieu naturel des relèvements de débit réservé constitueront avec les besoins de la collectivité les éléments de décision orientant le choix final d'un débit réservé.

J. Rietjens et $\mathrm{Ph}$. Gosse, dans leur communication « Les outils informatiques d'aide à la gestion intégrée de la ressource en eau dans une vallée: intérêt et limites ", décrivent les différents outils d'une politique de gestion de l'eau et les avantages qu'ils présentent au travers de réalisations auxquelles EDF a participé.

Ces différents outils se distinguent de la manière suivante: - les outils de diagnostic servant à caractériser usages et fonctions du milieu et aidant à la compréhension du fonctionnement des écosystèmes aquatiques,

- les outils d'orientation stratégique et de négociation, à partir de propositions en matière d'objectifs de qualité et de quantité pour le milieu et les usages permettant de tester des alternatives,

- les outils de suivi et de décision assurant un suivi du milieu en temps réel pour asseoir les décisions de gestion au quotidien.

Le champ de ces outils ne se limite pas aux aspects techniques, mais concerne aussi les aspects sociaux, organisationnels et économiques.

Au-delà des avantages que présentent ces outils informatiques et la démarche qu'ils constituent, nous devons rester lucides sur leurs limites. Les deux auteurs en soulignent les raisons majeures :

- complexité du fonctionnement des écosystèmes (difficulté de schématiser des intéractions complexes),

- problèmes liés aux mesures quant à leur fiabilité et au coût d'acquisition des données,

- champ d'investigation réduit, notamment concernant les contraintes d'ordre économique ou social.

En conséquence, il est indispensable de positionner avec justesse nos attentes par rapport à ces outils.

Les outils de diagnostic et de gestion ont été présentés de manière dissociée par simple commodité, mais ces outils sont étroitement imbriqués, comme le montre la communication de $\mathrm{Ph}$. Degardin, Ph. Lohest, L. Pereira-Ramos. L'évolution historique des réseaux de mesure et des redevances des Agences de l'Eau (redevance considérée comme outil de gestion) en témoigne.

En guise de conclusion, il convient de rappeler les remarques de ces auteurs qui considèrent, à juste titre, que les mesures sur le milieu et les usages sont des outils indispensables de connaissance, dont l'évolution est directement liée à celle des outils de gestion. La perspective d'une gestion intégrée doit inciter à " sortir du cours d'eau " pour s'intéresser au bassin versant, et à définir des objectifs pour utiliser au mieux outils de mesure et outils de gestion. 


\section{QUESTIONS AUX AUTEURS}

1) J. Eon et G. Merle - EDF Production Transport.

"Contribution de la méthode micro-habitats au choix du débit réservé ».

- Des démarches analogues ont-elles été engagées à l'étranger et en connaît-on les résultats ?

- Les études sont complexes; on ne pourra les effectuer sur chaque ouvrage (microcentrale par exemple). Peut-on imaginer avoir des valeurs régionales de débit réservé ? - Le choix d'un débit réservé ne passe-t-il pas a priori par une concertation locale : quelle espèce de poisson faut-il préserver?

- Où en est-on des études de débit réservé sur les rivières de seconde catégorie (les micro-habitats s'appliquant plus à des rivières de première catégorie) ?

Avant de répondre aux questions, je voudrais revenir en quelques mots sur la démarche (il s'agit plus d'une démarche qu'une "méthode EDF »), dans laquelle nous nous sommes engagées et qui fait l'objet de notre communication et sur nos motivations au premier plan desquelles figure le maintien des performances du parc hydroélectrique.

Le parc hydroélectrique présente pour EDF un outil essentiel pour l'équilibre du système production-transportconsommation. Le choix du débit réservé est un élément important de la gestion de ce parc qui comporte des enjeux économiques forts et évidemment aussi des enjeux écologiques.

J'ajoute que ce choix est aussi essentiel pour d'autres gestionnaires que les producteurs hydroélectriciens.

Pour ce qui concerne EDF, cette préoccupation du choix du débit réservé s'est manifestée dès 1982 dans une convention signée avec le ministère de l'environnement et le ministère de l'industrie. Et les premiers travaux sur ce sujet ont débouché sur l'appropriation, l'adaptation et le développement, de façon simultanée et coordonnée avec d'autres organismes publics de recherche comme le Cemagref et certaines universités, d'une méthode mise en œuvre aux USA quelques années plus tôt, la méthode des microhabitats.

$M$. Besème a rappelé les principes de la méthode et je n’y reviendrai pas

La méthode a fait l'objet de plusieurs études et il nous a semblé utile, et c'est ce que nous avons proposé à nos partenaires (organismes de recherche, administrations, agences de l'eau), de tirer profit de la situation créée par le renouvellement des titres administratifs de certaines chutes, accompagné d'une augmentation du débit réservé, pour mesurer in situ les réponses en terme de poissons et de biomasse et les relier aux évolutions d'habitats prévues par la méthode des micro-habitats.

Au stade où nous en sommes qui est celui de l'étape préliminaire qui consiste à proposer les débits qui seront expérimentés et suivis pendant 4 ans, on peut déjà tirer quelques enseignements et encouragements et constater l'intérêt de la démarche par:

- les échanges entre les différents partenaires,

- la mise au point de protocoles d'application de la méthode garantissant la qualité des résultats,

- une réflexion sur les critères d'interprétation des courbes (critères d'objectivisation et de calibration de la méthode), et sur leur utilisation en tant qu'outil d'aide à la décision (quelles espèces ?, quels stades de développement à privilégier?, quelle valeur d'habitat « acceptable»?),

- la nécessité d'une analyse de la sensibilité de la méthode.

\section{Réponses aux questions}

1) Des démarches analogues ont-elles été engagées à l'étranger et en connaît-on les résultats?
La méthode elle-même vient des USA. De nombreux développements sont en cours. Des échanges internationaux sont organisés : Symposium à Trondheim (Norvège) en 1994 sur le thème hydraulique et habitat, Colloque habitat-poissons à Lyon en décembre 1994.

L'AIRH a crée récemment une section "écohydraulique " et vient de participer à l'organisation (juin 1996) d'un colloque à Québec (Canada) sur le même sujet (\# 300 participants du monde entier et 130 communications).

On y relève de nombreuses communications sur la relation hydraulique et habitat et sur la part d'autres facteurs tels que la variabilité hydrologique, la sédimentation, la géomorphologie etc...

Les développements sur le perfectionnement de la méthode sont aussi nombreux: modèles $2 D$ et même $3 D$.

En revanche on y relève peu de sujets traitant de la relation habitat/biomasse (il est vrai que la morphodynamique n'est qu'une composante de la biomasse) ou de la mesure in situ de la réponse en terme de population à des modifications de régime hydraulique durable.

La France est sans doute de ce point de vue dans une situation particulière où du fait de sa législation un certain nombre d'autorisations d'exploitation à des fins énergétiques de chutes hydrauliques doivent être renouvelées et que ce renouvellement s'accompagnera nécessairement d'un relèvement du débit réservé (loi-pêche, code rural).

2) Les études sont complexes. On ne pourra pas les effectuer sur chaque ouvrage (microcentrales par exemple). Peuton imaginer avoir des valeurs régionales de débits réservés?

Les études sont complexes c'est vrai mais le problème en lui-même est complexe! Dans un premier temps au moins, il est difficile d'y répondre par des études trop sommaires quand il s'agit en fait de répondre à un objectif de prévision d'évolution suite à des modifications physiques.

Le travail déjà entrepris sur la méthode: procole de mise en auvre, modélisation hydraulique, logiciel de dépouillement permet de réduire les coûts.

Le coût de mise en auvre de la méthode, pour une station, est du même ordre de grandeur qu'une pêche électrique.

Une station est représentative des faciès d'écoulement dominant sur le tronçon de rivière étudié.

Le nombre varie en fonction de l'hétérogénéité des sites étudiés.

Beaucoup d'efforts ont été faits pour former les bureaux d'études et mettre à leur disposition un logiciel convivial.

Concernant la régionalisation, elle me paraît tout à fait envisageable en tous cas c'est aussi notre espoir. Le Cemagref a entrepris un travail de ce type sur le bassin de la Loire et celui du Rhône (notion d'écorégions où on peut mettre en évidence des comportements écologiques semblables). Une analyse de la réponse de l'habitat aux variations de débit basée sur la méthode des microhabitats. associée à une approche statistique des variabilités hydrauliques devrait aller dans cette voie. Nous espérons que l'investissement consenti par l'ensemble des partenaires dans cette démarche y contribuera.

3) Le choix d'un débit réservé ne passe-t-il pas a priori par une concertation locale?

La méthode doit être considérée comme un outil d'aide à la décision.

Elle propose dans une gamme de débits étudiés des réponses en capacité d'accueil potentiel liées aux exigences d'habitat de certaines espèces de poissons. Il s'agit là de répondre aux exigences de la loi-pêche, c'est-à-dire choisir un débit assurant la vie, la circulation et la reproduction des espèces. 
Le choix de l'espèce voire du stade de développement à privilégier dépend nécessairement d'une concertation locale. En fait, le choix doit résulter d'une réponse à une question préalable. Quel objectif pour la rivière?

Les outils que constituent les SDAGE et les SAGE prenant en compte une gestion équilibrée de la ressource en eau et intégrant aussi des objectifs généraux : objectifs de restauration de certaines espèces, objectifs socio-économiques (énergétique, industriel, touristique),... doivent donner des indications.

Le choix d'une espèce cible relève d'une simplification de la réalité. On peut s'appuyer sur des référentiels faunistiques régionalisés et raisonner en terme d'écarts par rapport aux potentialités régionales. La concertation locale peut alors aider à définir l'écart admissible dans les limites du respect des lois.

4) Où en est-on des études de débit réservé sur les rivières de deuxième catégorie?

\section{Réponse proposée par le Cemagref}

Cette extension nécessitait un investissement de recherche important, que le Cemagref a pris à sa charge. Cet effort a en partie été consenti pour développer des modèles biologiques pour les espèces de poissons électives de ces zones plus aval des cours d'eau. Une quinzaine d'espèces ont pu ainsi être décrites quant à leurs exigences fines d'habitat. La description physique de ces zones est parfois difficile lorsque le cours d'eau n'est plus praticable à pied. Là encore des solutions techniques ont été apportées grâce à des technologies modernes d'acquisition des profils de vitesse (ADCP Acoustic Dopler Current Profiler) et de repérage topographique (laser). La modélisation hydraulique est en revanche souvent plus aisée. Des applications concrètes ont été mises en auvre sur le Rhône, la Seine. la Garonne. Les premières validations sont encourageantes. L'interprétation des réponses faunistiques est plus délicate qu'en petit cours d'eau en raison de la plus grande complexité des cortèges faunistiques. L'expérience est également moindre.

Les équipes qui s'intéressent à ce thème se trouvent au Cemagref (Lyon. Aix. Paris) et à l'UniversitélCNRS de Lyon I en partenariat avec la CNR et l'Agence de l'eau. Elles travaillent en étroite collaboration.

2) Ph. Degardin - L. Pereira-Ramos et Ph. Lohest Agence de l'Eau Seine-Normandie.

"Les mesures sur le milieu et les usages de l'eau, révélatrices des outils de gestion $»$

- Pouvez-vous faire le point sur:

- les lacunes des mesures actuelles?

- les perspectives d'évolution des mesures et leur incidence sur les outils de gestion?

Les éléments de réponse apportés ici reposent sur l'expérience acquise par l'Agence de l'Eau Seine-Normandie dans le domaine des mesures. En effet, elle participe activement à l'acquisition et à la diffusion de connaissances et est impliquée chaque année dans plusieurs réseaux de mesures sur le milieu qui comptent environ :

- 200 postes pluviométriques et pluviographiques.

- 170 stations hydrométriques.

- 230 piézomètres.

- 360 stations d'analyses de la qualité des rivières.
- 150 stations de mesure de l'état piscicole des cours d'eau.

- 100 sites de mesure de suivi de la qualité bactériologique des eaux sur le littoral.

- 1000 analyses de qualité des eaux souterraines.

En outre. l'Agence de l'Eau réalise des mesures sur les pollutions issues des collectivités et des industries dans le cadre de son activité de perception des redevances.

L'appréciation des lacunes des mesures dépend bien évidemment des objectifs que l'on se fixe. On peut toutefois observer des domaines où les mesures sont négligées. II s'agit du suivi des eaux souterraines en comparaison de celui des eaux superficielles et d'une manière générale de ce qui relève de la quantité par rapport à la qualité. Pour illustrer ce dernier point, on peut citer le cas de la mesure du débit des cours d'eau en étiage qui pose des problèmes parfois insolubles. De même, les prélèvements d'eau pour l'irrigation sont aujourd'hui très mal connus. En outre, le suivi du milieu et des usages dans l'amont des bassins se révèle toujours délicat.

D'autres domaines souffrent également d'un manque. II s'agit du suivi des rejets qui est encore trop souvent limité aux rejets chroniques et ponctuels ou encore de l'accessibilité aux données recueillies.

Si l'on s'intéresse au cas particulier de la qualité des eaux superficielles, on constate que les mesures actuelles sont inadaptées pour apprécier la qualité globale des cours d'eau (les mesures physico-chimiques prédominent souvent). pour évaluer des flux de pollution ou encore apprécier les conséquences de pollutions épisodiques comme celles des rejets urbains de temps de pluie ou les pollutions diffuses d'origine agricole.

En outre, les quelques outils intégrateurs disponibles ne permettent pas de faire le lien entre les causes et les effets, ce qui favoriserait l'action en faveur des milieux aquatiques.

En terme d'évolutions prévisibles, on peut s'attendre à un développement des mesures, ce qui favorisera une amélioration des connaissances, dont la nécessité a été révélée dans les travaux de préparation du Schéma Directeur d'Aménagement et de Gestion des Eaux. Cet accroissement du volume des données ne pourra intervenir sans un travail concomitant sur la fiabilité des données, par des démarches d'assurance qualité par exemple. La mise au point d'un nouveau Système d'Evolution de la Qualité de l'Eau, objet d'une réflexion commune entre les Agences de l'Eau et le ministère de l'Environnement, permettra d'enrichir les objectifs de qualité. Et la généralisation de suivi d'indicateurs biologiques et le développement de la mesure en continu élargiront le champ de réflexion habituel et contribueront notamment à une meilleure gestion des ouvrages.

En conclusion. les outils de mesure présentent effectivement des lacunes mais celles-ci ne doivent pas masquer les satisfactions car ils demeurent un outil irremplaçable de connaissance. Les perspectives d'évolution sont à ce titre encourageantes. A l'avenir, les bassins versants dans leur globalité devront être pris en compte. Les mesures devront toujours veiller à pouvoir définir des objectifs quantifiables. compréhensibles et accessibles pour permettre la mise en auvre des outils de gestion équilibrée dont nous avons besoin.

\section{3) V. Ruban - L.C.P.C.}

"Prise en compte du phosphore des sédiments pour une meilleure gestion des écosystèmes aquatiques". - Application à la retenue hydroélectrique de Bort-les-Orgues (Massif Central). 
- Si la connaissance précise de la balance entre les apports externes et internes de phosphore est un préalable indispensable à toute opération de restauration de la qualité d'un plan d'eau, il reste néanmoins difficile d'apprécier les bénéfices que l'on peut attendre d'actions visant à limiter les relargages de phosphore à partir des sédiments.

- A partir des calculs de temps de réhabilitation, est-on en mesure de donner aux gestionnaires en charge des plans d'eau des préconisations précises en matière de gestion (faut-il agir au niveau des sédiments ; si oui, sur le stock? sur le relargage? laisser le temps faire son œuvre ?) Si non, quelles méthodes complémentaires faudrait-il concevoir?

La dégradation de la qualité des eaux due à un excès de substances nutritives (au premier rang desquelles le phosphore) et se traduisant par une prolifération de végétaux aquatiques (eutrophisation) affecte aujourd'hui bon nombre de rivières et de lacs naturels ou artificiels. De nombreuses mesures sont prises tant au niveau national qu'international pour restaurer la qualité de ces eaux douces superficielles. Ainsi, la Directive Européenne sur la collecte et le traitement des eaux résiduaires urbaines de mai 1991, définit des normes de rejet pour le phosphore et l'azote dans les "zones sensibles ", soumises à des risques d'eutrophisation.

Pour une meilleure gestion des écosystèmes aquatiques, il est nécessaire de prendre en compte le phosphore stocké dans les sédiments et susceptible d'être relargué : ce paramètre est cependant rarement considéré dans les calculs de temps de réhabilitation.

\section{Calcul du temps de réhabilitation}

Le temps de réhabilitation est un élément clef pour une bonne gestion des plans d'eau; ces calculs conduisent pourtant fréquemment à des échecs. En effet, les délais prévus pour qu'un lac retrouve des eaux de bonne qualité sont souvent supérieurs aux prévisions des modèles. Cela est lié au fait qu'une partie du phosphore est remobilisée à l'interface eau-sédiment et diffuse dans la colonne d'eau.

En règle générale, la prédiction de la réaction d'un plan d'eau aux apports nutritifs est réalisée par une étude statis-' tique de comportement de plans d'eau similaires. Le temps de réhabilitation est calculé à partir du temps de séjour du phosphore dans le lac :

$$
S[P]=[P]_{l} /[P]_{\text {in }}
$$

avec $[P]_{1}=$ masse annuelle de phosphore total dans le plan d'eau,

$[P]_{\text {in }}=$ apport annuel de phosphore total au plan d'eau.

Le temps de réponse est estimé à trois fois le temps de séjour du phosphore. Si le phosphore stocké dans les sédiments et susceptible d'être relargué n'est pas pris en compte, des différences parfois très importantes peuvent apparaître entre les prévisions et les résultats observés.

\section{Propositions de gestion des plans d'eau}

Sous réserve qu'il soit calculé de manière correcte, le temps de réhabilitation est un outil intéressant qui permet aux ges- tionnaires de plans d'eau de prendre des mesures adéquates. Ces mesures de traitement ne devront être employées que lorsque les apports de phosphore à la source auront été réduits. On distinguera alors deux cas, celui où un traitement complémentaire n'est pas nécessaire et celui où ce traitement s'avère indispensable.

- Pas de traitement.

Si le temps de réhabilitation est court, l'eutrophisation peu critique et le gestionnaire non désireux de faire des investissements supplémentaires, il n'est pas nécessaire d'effectuer un traitement. Le phosphore stocké dans les sédiments s'épuisera et si les apports à la source sont réduits, le lac retrouvera un état satisfaisant.

- Traitement nécessaire.

A l'inverse, en cas d'eutrophisation importante, d'une réduction insuffisante des apports à la source ou d'un temps de réhabilitation jugé trop long, un traitement pourra être envisagé. Il devra être adapté à la taille, aux caractéristiques et aux usages du plan d'eau. Ces traitements sont souvent très onéreux : parmi les plus répandus on peut citer :

- La désactivation du phosphore, qui a pour principe de fixer le phosphore par introduction dans l'eau de substances (sels de fer ou d'aluminium, chaux) provoquant la précipitation du phosphore.

- L'oxydation des sédiments. Le procédé Riplox consiste à injecter du nitrate de calcium ou du chlorure ferrique dans les sédiments afin d'éviter le relargage du fer et donc du phosphore. Cela est valable lorsque le fer contrôle le relargage du phosphore, ce qui est généralement le cas.

- Aération de l'hypolimnion. Une bonne oxygénation (injection d'oxygène, brassage) empêche le relargage du phosphore.

- Abaissement du niveau d'eau. Lors d'une vidange, les sédiments se trouvent exposés à l'air et on constate souvent une diminution du relargage après la remise en eau.

- Dragage. Cette technique est sans doute la plus efficace à long terme mais elle est très onéreuse et pose le problème du stockage des sédiments dragués.

- utilisation d'algicides. Le sulfate de cuivre est couramment employé pour combattre la croissance des algues.

- Biomanipulations. On modifie le réseau trophique par introduction d'espèces nouvelles (poissons, zooplancton) qui broutent les algues.

- Recouvrement des sédiments. Le sédiment est recouver d'une bâche plastique ou de particules (cendres volantes) visant à supprimer les échanges eau-sédiment.

\section{Conclusion}

La meilleure solution pour restaurer un plan d'eau consiste à réduire les apports à la source. Cependant, les résultats ne sont pas toujours ceux escomptés, notamment au niveau des temps de réhabilitation, car le relargage interne du phosphore n'est pas pris en compte.

Les mesures complémentaires de réhabilitation doivent être adaptées aux usages et aux caractéristiques de chaque plan d'eau, il n'y a pas de solution miracle.

Enfin, il faut noter que tous ces traitements ont un effet limité dans le temps (parfois quelques mois): de plus ils entraînent des perturbations du milieu naturel et sont côtteux. 


\section{EES MOYENS D'ACTION}

La gestion de l'eau dépend de trois instruments:

- l'instrument juridique et réglementaire,

- l'instrument économique et financier,

- I'instrument technique.

Seul l'instrument technique sera développé ci-après. Ce choix ne minimise pas l'importance des autres instruments qui appuient et encadrent les actions sur le terrain.

Les moyens d'action développés ci-après résultent du Schéma Directeur d'Aménagement et de Gestion des Eaux du bassin Loire-Bretagne. Ses préconisations générales sont transposables à d'autres bassins; il se différenciera des autres SDAGE par une hiérarchisation différente de ses objectifs.

La loi sur l'eau du 3 janvier 1992 a prescrit l'élaboration de SDAGE pour concilier les besoins de l'aménagement du territoire et la gestion équilibrée de la ressource en eau. Un seul SDAGE a été réalisé pour l'ensemble du bassin. Il a pour objet de fixer les orientations fondamentales de cette gestion. Celle-ci vise à assurer (article 2 de la loi sur l'eau) : - «la préservation des écosystèmes aquatiques, des sites et des zones humides,

- la protection contre toute pollution et la restauration de la qualité des eaux superficielles et souterraines et des eaux de la mer dans la limite des eaux territoriales,

- le développement et la protection de la ressource en eau,

- la valorisation de l'eau comme ressource économique et la répartition de cette ressource de manière à satisfaire ou à concilier, lors des différents usages, activités ou travaux, les exigences :

- de la santé, de la salubrité publique, de la sécurité civile et de l'alimentation en eau potable de la population,

- de la conservation et du libre écoulement des eaux et de la protection contre les inondations,

- de l'agriculture, des pêches et des cultures marines, de la pêche en eau douce, de l'industrie, de la production d'énergie, des transports, du tourisme, des loisirs et des sports nautiques, ainsi que de toutes autres activités humaines légalement exercées. "

Le SDAGE fixe les objectifs principaux pour une gestion harmonisée de l'eau, énonce des recommandations générales et particulières et arrête des objectifs de quantité et de qualité des eaux.

\subsection{Les objectifs fixés par le SDAGE}

Sept objectifs ont été ainsi définis :

\subsection{L'amélioration de la ressource pour l'alimentation en eau potable (AEP)}

La sécurité de l'alimentation en eau potable est une priorité. La fragilité de cette alimentation a été mise en évidence au cours des périodes de sécheresse de 1989 à 1993 : ressource peu abondante et/ou mauvaise qualité de l'eau (matières organiques, nitrates, phosphates, pesticides, métaux, pollution bactériologique).

Pour accroître la sécurité de I'AEP, le SDAGE préconise :

- d'améliorer la connaissance des nappes d'eau souterraine, d'éviter leur surexploitation et de les réserver en priorité à l'alimentation en eau potable,

- d'améliorer la qualité des eaux de surface susceptibles d'être potabilisées,
- de protéger les captages pour l'alimentation en eau potable,

- de moderniser et de fiabiliser les systèmes de traitement et de distribution d'eau potable, notamment par l'interconnexion.

\subsubsection{L'amélioration de la qualité des eaux superficielles}

Les progrès réalisés pour la réduction des pollutions toxiques et organiques carbonées ont été malheureusement compensés par une dégradation due à l'augmentation des nitrates et des phosphates, ce qui se traduit par une croissance de l'eutrophisation des cours d'eau.

Le SDAGE préconise :

- I'actualisation des objectifs de qualité des eaux superficielles en visant des classes de qualité supérieure pour les cours d'eau pour lesquels ces objectifs sont actuellement fixés en classe 3 ou hors classe. Ces futurs objectifs de qualité ne devront donc pas comporter de sections hors classe et les objectifs de classe 3 devront être strictement limités aux secteurs où un objectif plus ambitieux n'est pas réalisable, - la réduction de la pollution due aux rejets urbains, industriels et agricoles, par la mise à niveau des objectifs d'assainissement des collectivités et des industries, en application des directives européennes, notamment dans les domaines de la dénitrification et de la déphosphatation.

\section{I.3 La réhabilitation des cours d'eau}

Pour la restauration des écosystèmes aquatiques et des grands axes de migration des espèces piscicoles, le SDAGE préconise :

- la limitation des prélèvements en période d'étiage,

- le soutien des étiages,

- la restauration du lit des cours d'eau,

- la restauration de zones de frayères et d'habitats,

- des actions pour le retour des poissons migrateurs.

\subsubsection{La restauration des zones humides}

Ces zones ont une grande valeur écologique et assurent des fonctions de régulation importantes : auto-épuration et stockage d'eau participant à l'écrêtement des crues.

Pour leur restauration et leur préservation, le SDAGE préconise :

- le renforcement de la protection de ces zones et de leur suivi,

- des actions d'information et de sensibilisation des partenaires locaux et du public,

- l'arrêt progressif des extractions de matériaux dans le lit endigué et leur limitation dans le reste du lit majeur.

\subsubsection{La préservation et la restauration des écosystèmes littoraux}

\section{Le SDAGE préconise :}

- la mise en place d'indicateurs de qualité littoraux et d'un suivi du littoral,

- la réduction de la pollution bactériologique au droit de certains usages (baignade, pêche, conchyliculture,...) par un traitement adapté au niveau des stations d'épuration,

- la réduction des apports de nutriments, notamment d'azote, générateurs des phénomènes d'eutrophisation marine,

- la prise en compte de la pollution aquatique dans les projets d'aménagements littoraux,

- une protection accrue des estuaires. 


\subsubsection{La maîtrise des rejets agricoles}

L'intensification de l'agriculture est une cause importante de pollutions par les nitrates, phosphates et produits toxiques (pesticides), qui contaminent les nappes, les eaux superficielles et le littoral.

Pour réduire et maitriser ces rejets, le SDAGE préconise : - l'amélioration de la connaissance des prélèvements d'eau pour l'irrigation et une conduite raisonnée de l'irrigation, et si nécessaire la limitation des prélèvements,

- la réduction des rejets polluants des élevages,

- la contractualisation du service rendu par la profession pour l'épandage des boues d'épuration des eaux résiduaires urbaines ou industrielles,

- la mise en place de mesures agrienvironnementales.

\section{I.7 La maîtrise de l'urbanisation dans les zones inondables et l'amélioration de la protection des zones habitées contre les inondations}

Si les petites crues ont un rôle bénéfique pour les écosystèmes, les grandes crues peuvent mettre en danger la vie de nombreux riverains et occasionner des dégâts matériels importants.

Pour améliorer la sécurité des personnes et des biens, le SDAGE préconise :

- de mettre un terme à l'urbanisation dans les zones inondables à risque fort et dans les zones d'expansion de crue et de la limiter strictement ailleurs,

- l'amélioration de la protection des zones inondables déjà urbanisées, par la mise en œuvre d'une politique de restauration et l'entretien des digues de protection et du lit des cours d'eau.

Ce chapitre est illustré par la communication de H. Dumay intitulée « L'aménagement de la Loire à BrivesCharensac ». Elle relate le déroulement et le contenu de l'une des premières actions du plan « Loire Grandeur Nature ».

\section{- 5.2 Les préconisations générales}

Le rôle de l'eau et du réseau de fleuves et de nappes doit être considéré comme une infrastructure naturelle de l'aménagement du territoire.

L'arbitrage entre les usages se fera au niveau des SAGE, la seule priorité commune étant d'assurer, en période critique, les besoins vitaux de l'homme et des animaux.

Pour atteindre ses objectifs, le SDAGE comporte des préconisations par thème, dont les plus importantes sont les suivantes :

\subsubsection{Les milieux aquatiques continentaux et littoraux}

- la reconnaissance de l'utilité collective des milieux naturels,

- la limitation de la construction d'ouvrages dans le lit des cours d'eau,

- la limitation de la création de plans d'eau,

- la détermination au niveau de chaque ouvrage du débit minimal biologique, afin d'améliorer les débits réservés et limiter les effets des éclusées,

- la création de structures permanentes d'entretien des rivières,

- lorsque cela est nécessaire, la maîtrise foncière des rives par les collectivités locales pour mieux les entretenir, aménager des accès publics et, au besoin, créer des zones de transition végétalisées entre le cours d'eau et les cultures,

- pour les eaux marines: la détection des phytosanitaires et des mesures de l'eutrophisation,
- pour les eaux souterraines : l'acquisition de données, la modélisation du fonctionnement des aquifères, la gestion patrimoniale de la ressource,

- pour les zones humides: la reconnaissance de leur valeur fonctionnelle, l'arrêt de leur régression et la réhabilitation d'anciennes zones humides par la mise en cuvre d'une politique de préservation et de gestion, incluant des mesures agrienvironnementales et l'inscription de ces zones dans les documents d'urbanisme.

A. Amezal, auteur de « Pour une stratégie de gestion équilibrée des milieux aquatiques et humides du bassin Seine-Normandie », explique la démarche de cette Agence vis-à-vis de la politique d'aménagement de rivières.

La préparation du $\mathrm{VII}^{\mathrm{e}}$ programme d'intervention (19972001) a conduit à la réalisation d'un bilan de cette politique. Il en est ressorti de nouvelles orientations distinguant nettement les opérations multiusages ( « aménagements de gestion équilibrée »), des opérations à vision fragmentaire. La reconquête, la protection et la valorisation des milieux aquatiques et humides doivent motiver toute intervention pour être subventionnable par l'Agence Seine-Normandie.

La gestion des milieux aquatiques continentaux est parfaitement illustrée par la communication de F. Degardin intitulée "Réflexions sur l'usage des vallées et le fonctionnement des cours d'eau». Si, dans une première phase, ce travail étaye les enjeux écologiques traités précédemment en insistant particulièrement sur la nécessaire prise en compte des phases d'écoulement, il se positionne en terme d'action quand il décrit les conduites possibles en matière de gestion des cours d'eau et des vallées, largement illustrées par des exemples.

\subsubsection{Les prélèvements}

- pour la protection des points de prélèvements d'eau potable, l'étude de faisabilité des périmètres de protection dans un délai de 3 ans après l'approbation du SDAGE,

- dans le même délai, l'étude de la sécurité des distributions publiques d'eau potable,

- la fixation d'un objectif de pertes dans les réseaux, qui ne soit pas supérieur à $20 \%$.

- l'encouragement aux économies d'eau par les industriels et les agriculteurs, en diffusant, notamment à ces derniers, les résultats des secteurs de référence irrigation.

\subsubsection{La qualité des eaux}

- la révision des cartes d'objectifs de qualité des eaux superficielles dans le respect des objectifs fixés aux points nodaux (voir préconisations particulières), en éliminant la hors classe et la classe 3 ,

- la fixation d'objectifs de qualité pour les lacs et les étangs,

- la réduction de la pollution phosphatée, la concentration en phosphore total étant ramenée à $0,2 \mathrm{mg} / \mathrm{l}$ dans un délai de 10 ans,

- l'organisation de la lutte contre les phytosanitaires et les métaux lourds par des actions ciblées dans le bassin,

- la réduction et la gestion des flux d'azote émis par l'agriculture, et l'aménagement de barrières au transfert des pollutions (zones humides),

- le contrôle des rejets urbains de temps de pluie et de temps sec.

- l'aménagement des barrages pour réduire les impacts des vidanges (pièges à sédiments) et restituer en tout temps des eaux de qualité compatible avec les objectifs généraux,

B. Remini, J.M. Avenard et A. Kettab, dans leur communication intitulée «Evolution de l'envasement dans la partie centrale de la retenue d'un barrage " ont étudié, à partir d'une dizaine de retenues de barrages en Algérie, le comportement des sédiments entrants dans différentes zones des 
retenues afin de fiabiliser la technique du soutirage pour prolonger la durée de vie des ouvrages.

- la décontamination des eaux rejetées à proximité des plages et des zones conchylicoles.

\subsubsection{La sécurité face au risque d'inondations}

- l'arrêt de l'urbanisation dans les zones inondables, notamment en interdisant dans les documents d'urbanisme les constructions dans les zones à risque fort, définies dans les atlas des zones inondables et en élaborant des plans de protection contre les risques naturels.

- l'entretien des cours d'eau, des levées et des digues existantes.

- la préservation des champs d'expansion des crues.

- la maîtrise du ruissellement par l'aménagement du foncier agricole.

- l'écrêtement des crues par l'utilisation des ouvrages existants,

- le renforcement de l'annonce des crues.

B. Lancelot, dans sa communication intitulée « Le rôle des Agences de l'Eau face aux inondations ", expose ce que pourrait être la politique de I'Agence de I'Eau Seine-Normandie en matière d'inondations.

\subsubsection{La navigation et la baignade}

- l'évaluation des impacts sur le régime des eaux, des infrastructures existantes inutiles à la navigation et. si besoin est, leur modification.

- la préservation, au moment de la conception de nouveaux ouvrages, de la possibilité d'une navigation de plaisance adaptée aux cours d'eau.

- le maintien de la salubrité des lieux de baignade en rivière.

\subsubsection{Les extractions de matériaux}

- l’arrêt des extractions de matériaux dans les lits endigués et la limitation de ces extractions dans les autres zones inondables.

- l'insertion dans les schémas départementaux de carrières de conditions mises aux nouvelles extractions, tendant à préserver les zones humides et les captages.

- I'incitation des maîtres d'ouvrages publics à utiliser des matériaux de substitution aux granulats alluvionnaires.

\subsubsection{La pêche en eau douce et en mer}

- l'interdiction de la pêche amateur ou professionnelle, en mer et en rivière, de saumon de souche Allier, tant que les stocks ne seront pas reconstitués.

- la suppression des obstacles à la remontée des poissons migrateurs.

- des actions sur le bouchon vaseux de l'estuaire de la Loire pour rétablir une teneur en oxygène dissous supérieure à $3 \mathrm{mg} /$ litre.

- la réhabilitation et la protection des frayères à brochets,

- le contrôle de la qualité sanitaire des gisements naturels de coquillages.

\subsection{Préconisations particulières}

\subsubsection{Objectifs aux points nodaux}

Pour atteindre les objectifs généraux du SDAGE, celui-ci a fixé des objectifs de qualité de l'eau et de débit en 84 points nodaux du bassin. II s'agit de lieux particulièrement importants du bassin, le plus souvent situés à des confluences. embouchures et résurgences, permettant de contrôler l'évolution de la qualité de l'eau et des débits des cours d'eau.

Dans les parties sensibles du littoral (baies), le SDAGE définit des zones nodales, permettant la prise en compte de toutes les pollutions (cours d'eau et rejets directs).

\subsubsection{Objectifs de qualité aux points nodaux}

Des objectifs de qualité sont fixés pour chaque point nodal sous forme de concentrations limites pour les paramètres essentiels (matières organiques, nitrates, phosphore, chlorophylle, pesticides, métaux) relatifs aux usages de l'eau et au développement du milieu naturel.

$90 \%$ des mesures effectuées pour chaque paramètre en un point nodal doivent respecter les objectifs de qualité, à l'exception des mesures concernant les pesticides et les métaux qui doivent toutes satisfaire aux objectifs de qualité.

Les cartes d'objectifs de qualité des eaux superficielles devront être révisées pour tenir compte des objectifs de qualité fixés aux points nodaux.

Des objectifs de qualité peuvent être fixés pour les eaux marines, dans les parties des zones nodales où s'exercent certains usages, tels que parcs conchylicoles, baignades ou pêche à pied.

\subsubsection{Objectifs de débit aux points nodaux}

Les objectifs de débit aux points nodaux sont définis par référence à trois niveaux de seuil prenant en compte les usages de l'eau et le fonctionnement du milieu naturel :

- le débit objectif d'étiage.

- le débit seuil d’alerte.

- le débit d'étiage de crise.

Le débit objectif d'étiage (DOE) est le débit d'étiage en dessous duquel l'ensemble des usages n'est plus compatible avec le milieu aquatique et l'écosystème.

Ce débit est défini par référence au débit moyen mensuel minimal de fréquence quinquennale (QMNA5) et doit servir de référence pour l'exercice de la police de l'eau pour accorder des autorisations de prélèvement et de rejet.

Lorsque le DOE est inférieur ou égal au QMNA5. l'ensemble des usages actuels peut être satisfait sans porter atteinte au développement normal du milieu naturel. En revanche, lorsque le DOE est supérieur au QMNA5, la satisfaction de l'ensemble des usages n'est pas compatible avec le développement normal du milieu naturel. Il est alors nécessaire, pour éviter de porter gravement atteinte à celuici, soit d'augmenter le débit d'étiage par des ouvrages à définir, soit de réduire en certaines périodes les prélèvements.

Le débit seuil d'alerte (DSA) est le débit moyen journalier à partir duquel des mesures sont prises par les préfets pour la réduction des prélèvements. La limitation de ceux-ci est d'autant plus importante que le débit moyen journalier est faible.

Le débit d'étiage de crise (DCR) est le débit moyen journalier en dessous duquel l'alimentation en eau potable et la survie des espèces les plus intéressantes du milieu ne sont plus garanties. Les prélèvements pour les autres usages doivent alors être arrêtés.

\subsubsection{Nappes remarquables}

Les nappes souterraines constituent une ressource importante et de grande qualité, parfois surexploitée. Pour que cette ressource reste pérenne, la protection et la gestion de ces nappes doivent être maîtrisées.

Le SDAGE a répertorié 6 nappes intensément exploitées à une échelle régionale.

Des procédures devront être engagées pour le classemen de ces nappes en zone de répartition, pour celles qui n'ont pas encore fait l'objet d'un décret de classement. Le SDAGE préconise la mise en place de structures de gestion de ces nappes. 
Les nappes naturellement protégées et délivrant une eau d'excellente qualité doivent être considérées comme stratégiques. Des mesures de protection de cette ressource doivent être prises, car les prélèvements sont importants, alors que leur réalimentation est lente.

Un exemple:

La nappe des calcaires de Beauce, au nord de la Loire, qui couvre une superficie d'environ $9000 \mathrm{~km}^{2}$ et située moitié dans le bassin Loire-Bretagne et moitié dans le bassin SeineNormandie, est très sollicitée par des prélèvements pour l'agriculture. Le SDAGE préconise un vaste programme d'acquisition de données pour mieux connaître le fonctionnement de cette nappe et améliorer sa gestion. Quatre points nodaux concernent cette nappe et des objectifs de débit ont été déterminés, ainsi que trois niveaux de seuil, le plus bas correspondant au DCR des cours d'eau.

Cette nappe doit être classée en totalité en zone de répartition et la mise en place d'une structure de gestion est préconisée.

En conclusion, le SDAGE s'inscrit dans une stratégie de développement durable basée sur la prévention (maîtrise des prélèvements et des rejets, maîtrise de l'occupation du sol dans les zones inondables) et la protection. Ainsi, la pérennité de la ressource en eau, tant en quantité qu'en qualité, pourra être préservée et les effets des risques naturels (étiage, crues) réduits, ainsi que l'importance des dommages en résultant.

Les SAGE doivent reprendre les objectifs généraux et les objectifs qualitatifs et quantitatifs du SDAGE, et définir les modalités de gestion pour les atteindre. L'élaboration de SAGE est indispensable pour la mise en cuvre dans les sous-bassins des orientations et des objectifs fixés par le SDAGE.

La mise en œuvre de cette politique à travers les SAGE devrait permettre aux cours d'eau du bassin de retrouver un équilibre plus naturel et plus écologique, pour le plus grand bénéfice des riverains et de tous les habitants du bassin.

\section{1) H. Dumay - B.C.E.O.M.}

"L'aménagement de la Loire à Brives-Charensac ».

- Pouvez-vous nous expliquer comment s'est effectuée localement la concertation et quelle a été la réaction de la population face à cette procédure peu habituelle ? - Si cet aménagement résoud les problèmes des inondations à Brives-Charensac, que peut-on dire notamment en aval: $y$ a-t-il un risque ? Comment est-il pris en compte?

- Les travaux sont désormais quasiment terminés. A-t-on constaté des nuisances importantes ?

\section{PRÉALABLE}

Il y a deux parties dans la question:

1. Impact hydraulique de l'aménagement sur l'aval en terme d'aggravation du risque?

2. Ce nouveau concept d'aménagement recueille-t-il l'adhésion des populations riveraines et des diverses associations et collectivités?

Pour répondre aux questions posées, il faut préciser en préalable en quoi ce projet constitue une nouvelle solution. un nouveau concept d'aménagement :

- nouveau concept hydraulique de protection.

- association d'une action de protection à une action de valorisation du milieu.

\section{Comparaison des deux concepts de protection}

\begin{tabular}{|c|c|c|}
\hline & L'endiguement & La solution proposée \\
\hline $\begin{array}{l}\text { Protection } \\
\text { contre les } \\
\text { crues }\end{array}$ & $\begin{array}{l}\text { - élève les } \\
\text { niveaux de } \\
\text { crue } \\
\text { - supprime les } \\
\text { zones d'expan- } \\
\text { sion } \\
\text { - accroît le } \\
\text { risque } \\
\text { (rupture...) } \\
\text { tout en désen- } \\
\text { sibilisant }\end{array}$ & $\begin{array}{l}\text { - rabaisse les } \\
\text { niveaux de crue par } \\
\text { une action d'appro- } \\
\text { fondissement du lit et } \\
\text { de décaissement des } \\
\text { risbermes } \\
\text { - minimise le risque } \\
\text { par une inondation } \\
\text { lente et progressive } \\
\text { sans vitesse exces- } \\
\text { sive }\end{array}$ \\
\hline $\begin{array}{l}\text { Valorisation } \\
\text { du milieu }\end{array}$ & $\begin{array}{l}\text { - encage la } \\
\text { rivière } \\
\text { - Ia coupe de } \\
\text { son milieu } \\
\text { urbain }\end{array}$ & $\begin{array}{l}\text { - favorise les } \\
\text { relations ville-fleuve } \\
\text { en supprimant tout } \\
\text { obstacle (digue) } \\
\text { ouvre la ville vers } \\
\text { le fleuve }\end{array}$ \\
\hline
\end{tabular}

\section{Réponse à la question 1}

\section{Au début du projet}

Le projet a été non seulement accepté mais choisi par les riverains.

En effet, un concours préalable a été lancé dans le cadre d'un appel d'offre européen.

Trois bureaux d'études dont BCEOM ont été retenus pour présenter leurs projets.

La présentation de ce projet a donné lieu à une exposition publique à grande échelle qui a duré 10 jours.

Entre autres, BCEOM présentait les plans où apparaissaient les zones mises hors d'eau pour une crue type 1980. c'est-à-dire 100 ans mais montrait également les zones où subissaient des zones d'inondation urbaines.

Malgré cela, pour les raisons évoquées en préalable, les Brivois ont opté pour cet aménagement.

C'est en ceci que le projet est novateur car bâti sur un nouveau concept qui a permis une évolution des mentalités et du concept d'aménagement basés sur:

- "sécurité absolue ": oui, en acceptant malgré tout certaines nuisances pour des crues exceptionnelles inévitables.

- protection absolue non : car ça n'existe pas.

Prise de conscience mais surtout prise de responsabilité des populations : "vivre avec les crues» et ne pas les ignorer.

\section{Au fil du temps}

Après le concours

- Phase d'affinage et de procédures administratives qui s'est déroulée en concertation avec la commune et les associations.

- Nombreuses réunions publiques d'information sur les 3 communes de Brives-Monteil-Chadrac dans le cadre d'une démarche de communication (une dizaine de réunions en 2 mois).

- Ouverture d'une agence à Brives (avec exposition permanente de plans et photomontages) qui recevait le public et les associations.

- Enquête D.U.P. avec adhésion:

- de la ville.

- des associations de protection de la nature.

- quelques négociations bien entendu auprès d'entreprises pour tirer le meilleur parti des travaux.

\section{En cours de travaux}

- Action de communication permanente pour expliquer le projet, les travaux. Parution d'un journal de chantier en 4000 exemplaires tous les 2 mois.

- Comité de suivi environnemental du projet qui regroupe l'entreprise, le maître d'ouvrage et les associations pour prévenir et répondre aux problèmes posés. 
- Les craintes de nuisances sonores, de pollution par les poussières, de risques liés au déroctage par tir de mines ont très vite été apaisées. Malgré tout, quelques nuisances bien acceptées par la pollution qui croit au projet.

Les projets constituent d'ailleurs un spectacle journalier (tir de mines...).

- Travaux à sec dans le lit par isolement de bandes longitudinales endiguées. Quelques nuisances constatées fin juillet sur l'aval par une pollution en MES par des argiles rouges au moment du déplacement d'une digue.

- Peu de nuisances liées à la circulation qui se fait sur les digues dans le lit.

\section{Réponses à la question risque sur l'aval}

D'après le concept même du projet :

- rabaissement des niveaux de crue mais reconstitution des zones de débordement. Cela fait que globalement les volumes de laminage sont maintenus. Pour certains secteurs uniquement les vitesses d'écoulement sont augmentées localement de 10 à $15 \%$.

- la mise en auvre du modèle en régime transitoire permet d'ailleurs de répondre très précisément à cette question.

- Il n'y a pas d'accélération significative de la propagation de la crue sur l'aval.

- Le débit de pointe:

- n'est pas modifié pour une crue décennale,

- est augmenté de quelques $\mathrm{m}^{3} / \mathrm{s}$ pour une crue centennale.

Ces valeurs peu significatives du fait de leurs faibles modifications permettent de conclure qu'il n'y a pas d'impact sur l'aval.

La seconde partie de la question «comment le risque induit par le projet sur l'aval est-il pris en compte? "n'a pas lieu d'être compte tenu de l'absence d'impact.

\section{2) A. Amezal - Agence de l'eau Seine-Normandie.}

"Pour une stratégie de gestion équilibrée des milieux aquatiques et humides du bassin Seine-Normandie ».

- Pouvez-vous nous exposer les grandes lignes de la politique d'aménagement de rivières du $\mathrm{VII}^{\mathrm{e}}$ programme ?

- Que proposez-vous pour augmenter le nombre de maîtres d'ouvrage concernés par cette politique?

\section{Réponse à la question 1}

L'Agence de l'Eau Seine-Normandie a engagé une réflexion sur sa politique en matière d'aménagement de rivières au cours de son $\mathrm{VI}^{e}$ programme d'intervention (1991-1996).

Un bilan des actions menées en 1995 a mis en évidence notamment :

- une évolution nette vers des opérations dites « douces » de restauration, avec une demande accrue pour des travaux d'entretien,

- des travaux hydrauliques, bien qu'en régression, importants,

- une majorité des opérations motivées par une amélioration des conditions d'écoulement.

- des protections de berges en milieu rural trop importantes,

- un nombre insuffisant de maîtres d'ouvrage.

Afin d'intervenir à ces différents niveaux, une évolution de la politique s'avérait nécessaire.

Par ailleurs, une évolution des mentalités s'est produite avec la loi sur l'eau et le Schéma d'Aménagement et de Gestion des Eaux du bassin Seine-Normandie. De plus, deux études relatives aux zones humides, l'une sur les aspects fonctionnels, l'autre sur leur intérêt économique ont permis d'apporter des arguments supplémentaires à cette évolution. Parallèlement, l'évaluation des politiques publiques en matière de zones humides a mis en évidence une dégradation importante de ces zones.
Au vu de ce constat, de nouveaux objectifs d'intervention ont donc été définis pour le VII programme (1997-200I) avec une politique à la fois plus restrictive, par rapport aux actions précédentes, orientée vers une gestion équilibrée des rivières (en excluant tous travaux à objectif unique d'amélioration des conditions d'écoulement, ou tous travaux dégradant le milieu naturel), et à la fois plus élargie, en intégrant les zones humides.

Cette politique de gestion équilibrée des milieux aquatiques et humides dont l'objectif est la reconquête, la protection, la valorisation de ces milieux, doit viser à :

- satisfaire plusieurs usages et non plus un seul.

- s'inscrire dans une vision globale à l'échelle du bassin.

- intégrer les zones humides,

- avec des travaux uniquement de mise en valeur du lit mineur et majeur.

Le VII programme d'attribution des aides (1997-2001) de l'Agence de l'Eau Seine-Normandie doit donc permettre d'apporter des aides :

- aux études et aux travaux de réhabilitation et d'entretien,

- à la mise en place d'assistance technique (cellule d'assistance technique à l'entretien des rivières ou de zones humides, garde-rivière ou zones humides).

- aux acquisitions foncières de zones humides " prioritaires" ou de bandes rivulaires,

- à la contractualisation à la gestion, en finançant la mise en auvre de plans de gestion conformes aux objectifs de l'Agence,

- aux opérations relatives à l'information et au paysage, si elles sont associées aux interventions précitées,

- à la mise en place de postes nouveaux d'animateur en milieu rural.

Réponse à la question 2

Le nombre des maîtres est insuffisant. $40 \%$ du linéaire du bassin Seine-Normandie est couvert. En effet, sur $33000 \mathrm{~km}$ de linéaire de rivières, $20000 \mathrm{~km}$ sont laissés à l'abandon. Sur les $13000 \mathrm{~km}$ restant, 300 regroupements de collectivités environ réalisent des travaux de restauration et sur $50 \%$ seulement (soit $6500 \mathrm{~km}$ ) un entretien régulier est assuré.

Différentes actions sont donc envisagées à partir de 1997 pour remédier à cette situation.

Tout d'abord des actions d'information et de communication, avec plaquettes, films, journées d'information, seront réalisées. Les taux de subvention pour les travaux de restauration et d'entretien ainsi que pour la création d'un poste de garde-rivière seront majorés de $10 \%$. Des aides nouvelles seront attribuées pour :

- l'acquisition de bandes rivulaires et de zones humides,

- la contractualisation à la gestion.

- l'information et le paysage.

Par ailleurs, des aides importantes seront accordées à la mise en place de postes nouveaux d'animateur "milieu rural ». Ces derniers devront notamment favoriser la cohésion entre divers acteurs dans le domaine de l'eau, animer et conseiller les maîtres d'ouvrage, voire favoriser leur création. Parallèlement, l'Agence de l'Eau Seine-Normandie envisage de rechercher des relais locaux auprès de certaines structures existantes (collectivités territoriales, associations...) qui pourraient avoir un rôle de prospection et d'animation pour la recherche de maîtres d'ouvrage.

\section{3) F. Degardin - C.E.R.T.U.}

«Réflexions sur l'usage des vallées et le fonctionnement des cours d'eau

- Compte tenu du nombre important de cours d'eau recalibrés, pouvez-vous nous exposer succinctement l'impact du recalibrage sur le fonctionnement du cours d'eau ?

- Quelles sont les voies que vous proposez en matière d'arbitrage de conflits d'usages ? 


\section{Réponse à la question 1}

Le recalibrage vise à diminuer voire à supprimer les débordements ; à l'opposé il développe les hautes eaux actives, c'est-à-dire celles où les matériaux du fond et des berges sont mobilisés. Les effets du recalibrage sur le fonctionnement du cours d'eau sont donc les suivants (voir tableaux I et II de la communication) :

- multiplication des jours de hautes eaux actives et diminution forte sinon suppression des jours de débordement.

- augmentation de la hauteur d'eau et de la vitesse, et souvent aussi de la turbulence, des écoulements à pleins bords,

- monopole ou prédominance marquée des phases de creusement, donc abaissement du fond du lit mineur.

- diminution des alimentations de la nappe sous filtre naturel par les débordements et multiplication des situations de drainage de la nappe,

- disparition ou forte réduction des annexes et des zones humides en lit majeur.

Au total le recalibrage entraîne le creusement du lit mineur, souvent de façon intense, l'abaissement global de la nappe phréatique et l'assèchement des parties aquatiques ou humides du lit majeur. En outre en diminuant les débordements, il accélère la propagation des crues (exemple de la Haute Moselle entre Epinal et Toul, distantes de $70 \mathrm{~km}$, où le temps de propagation moyen est passé de 24 heures à 16 heures).

Il faut également signaler certains effets induits du recalibrage, notamment l'augmentation des dommages en cas d'inondation exceptionnelle. En effet, comme cela se passe derrière des digues, la raréfaction des débordements entraîne l'accumulation de biens, d'activités et de productions agricoles, industrielles et tertiaires dans le fond de vallée ; le fonctionnement exceptionnel du lit majeur entraîne alors des dommages non négligeables.

2) Propositions pour l'arbitrage des conflits d'usage.

Il est assez difficile de proposer des règles pour l'arbitrage des conflits d'usage sur les cours d'eau, les vallées et les bassins versants, si ce n'est la nécessité de pratiquer la concertation. Le nombre des acteurs (collectivités locales. services de l'Etat, agences de l'eau, propriétaires, exploitants de toutes sortes, associations d'usagers) et la diversité des usages suffisent à justifier ce dialogue même s'ils ne le facilitent pas. La simple écoute réciproque des besoins, des opinions, des réclamations et des suggestions des uns et des autres suffit parfois au règlement des problèmes (expérience personnelle d'un conflit entre irriguants de maïs et producteurs de pins le long de l'Escamat à Sabres dans les Landes). Pour une gestion plus complète et plus complexe de vallées ou de bassins, des procédures existent et des structures peuvent être utilisées ou créées : c'est le cas notamment des SDAGE et des SAGE.

Par ailleurs l'expérience tend à montrer l'intérêt de considérer les conflits d'usage à l'échelle territoriale la plus vaste possible: un problème local tend à se durcir lorsque quelques interlocuteurs focalisent leurs positions sur un espace réduit; au contraire il sera relativisé sur un secteur de référence plus étendu et par des acteurs plus divers et souvent plus puissants. Par exemple, pour une implantation d'activités, il faudra passer de la zone en fond de vallée à l'ensemble d'un territoire communal (exemple du projet de zone industrielle à Fains-Véel dans la Meuse, détaillé dans la communication).

Un autre exemple de résolution de conflit d'usages par la prise en considération d'un espace plus vaste et d'acteurs plus nombreux, est celui de la vallée de la Moselle à la limite des Vosges et de la Meurthe-et-Moselle. Un secteur d'une dizaine de kilomètres a conservé un état relativement naturel avec un lit mineur mobile, des débordements très fréquents, des noues et une forêt de rive développés mais aussi un gisement important de matériaux alluviaux de très bonne qualité. Un conflit est apparu entre les projets d'exploitation de gravier en lit majeur et le maintien d'une zone humide à forte biodiversité et de réserves pour l'alimentation en eau potable et le stockage des crues. Les tentatives d'arbitrage au niveau des syndicats intercommunaux et des deux commissions départementales des carrières n'ont pas abouti durablement. Le passage au niveau de la région et du bassin a permis le montage d'une solution acceptable par la plupart des propriétaires et des usagers : le Conservatoire Naturel Régional de Lorraine, subventionné par l'Agence de l'Eau Rhin-Meuse et par l'Union Européenne, acquiert les terrains convoités et les loue en convention spéciale aux exploitants actuels. Le changement d'échelle spatiale a permis de prendre en compte la dimension réelle des usages en conflit et d'impliquer des acteurs efficaces dans le financement des solutions.

4) B. Remini - Université de Blida, J.M. Avenard Université Louis Pasteur Strasbourg, A. Kettab - ENP Algérie.

"Evolution de l'envasement dans les retenues utilisant la technique de soutirage ».

- L'étude de ce phénomène est intéressante. Doit-on en tirer la conclusion qu'il n'y a aucun moyen d'empêcher l'inversement des retenues dans cette partie centrale qui est la plus importante?

\section{Réponse à la question}

Effectivement, la partie centrale est la plus importante de la retenue et reçoit des quantités considérables en sédiments. La technique du soutirage peut contribuer à la réduction de l'envasement. Le rendement le plus important, enregistré au barrage d'Ighil Emda (Algérie), est de $55 \%$ durant la période 1984-1992 (55\% des apports solides totaux entrant dans la retenue ont été soutirés par les vannes de fond).

D'après les derniers résultats que nous avons obtenus (résultats non encore publiés), ce rendement peut être amélioré pour atteindre la valeur de $70 \%$ (limite), le reste (30\%) ne peut pas être évacué par cette technique et reste dans la partie centrale: généralement cette quantité est constituée de sable et limons, la technique du soutirage intéresse surtout les particules fines situées dans la partie basse de la retenue. Aujourd'hui, le dévasement de la partie centrale n'est possible que par la technique de dragage (qui coûte cher) ou bien par la réalisation d'un barrage de chasse à l'amont, capable de produire des ondes artificielles très puissantes et de faire avancer les sédiments vers les vannes de fond.

Il est à signaler que ce phénomène est spectaculaire dans la région du Maghreb; plus de $100 \mathrm{Mm}^{3}$ de sédiments se déposent annuellement dans les barrages.

En Algérie on perd, chaque année, un nombre de $20 \mathrm{Mm}^{3}$ par les dépôts successifs de sédiments dans les réservoirs.

\section{5) B. Lancelot - Agence de l'Eau Seine-Normandie.} "Le rôle des Agences de l'Eau face aux inondations ".

- Quel type de redevances peut-on imaginer pour permettre aux Agences de l'Eau d'intervenir de façon significative dans le domaine de la prévention des inondations ?

\section{Réponse à la question}

La redevance pourrait être celle intitulée "Modification du régime des eaux " du décret 66.700 du 14 Septembre 66 relatif à la loi sur l'eau de 1964. 
Toutefois, à la différence des autres redevances des agences de l'eau. l'assiette de celle-ci reste à définir.

L'Agence de l'Eau Seine-Normandie a imaginé, dans un souci de simplicité de mise en auvre de la perception de cette redevance, que cette assiette pourrait :

- être basée sur la principale cause des inondations pluviales en milieu urbain: l'imperméabilisation des sols ;

- toucher les principaux bénéficiaires des travaux de protection : les populations urbaines.

Aussi, celle-ci toucherait-elle les propriétaires publics et privés de surfaces imperméabilisées ou dont l'occupation des sols aggraverait les dommages liés aux inondations. Les redevables seraient alors des maîtres d'ouvrages publics ou privés, propriétaires de surfaces imperméabilisées (ou aux facteurs aggravant les dommages) supérieures à une certaine superficie qui pourrait être un hectare.

Un système de prime serait envisagé. Il serait fonction de l'effet des aménagements des terrains « redevables" sur la maîtrise du ruissellement. de l'effort engagé par les maîtres d'ouvrages pour préserver les zones d'expansion des crues ou pour rendre compatible le tvpe d'occupation du sol avec les inondations.

Les objectifs que poursuivraient l'Agence de l'Eau SeineNormandie, si une telle redevance se mettait en place, seraient :

- pousser les maîtres d'ouvrage à une gestion globale des vallées, conformément à ce qui est inscrit dans le SDAGE du bassin :

- favoriser la cohérence des actions au niveau du bassin en terme de prévention prioritairement, mais aussi de protection dans la mesure où les travaux respectent l'équilibre du milieu naturel:

- ne pas aggraver le risque d'inondation en milieu déjà urbanisé, tout en veillant à protéger le milieu naturel.

Les conditions auxquelles seraient soumis les travaux de protection faisant l'objet d'une aide financière de la part de I'Agence de l'Eau Seine-Normandie, seraient :

- qu'ils concernent des zones déjà construites (et donc ne favorisant pas l'urbanisation des zones inondables qui, quels que soient les travaux, le seront toujours, le risque nul n'existant pas):
- qu'ils soient conçus au sein d'un schéma global de bassin (faisant suite à une étude des risques du bassin avec définition de l'impact des aménagements prévus sur l'ensemble du bassin):

- qu'ils se fassent parallèlement à des mesures sur l'occupation des sols visant à limiter le ruissellement ou favorisant l'expansion des crues à l'amont des agglomérations :

- avec un maître d'ouvrage adapté du type syndicat de rivière intercommunal ou entente interdépartementale, et/ou avec l'avis de la Communauté Locale de l'Eau lorsqu'elle existe :

- que leurs effets ne contribuent pas à la banalisation du milieu aquatique (lit mineur plus annexes hydrauliques) tel que cela peut arriver suite à des recalibrages de cours d'eau ou à des coupures de méandres...

L'Agence favorisera par contre la prévention, c'est-à-dire tout ce qui évite les dommages sans éviter forcément les inondations (qui peuvent être bénéfiques pour le milieu naturel) :

- la connaissance du risque (cartographie, modélisation, étude d'incidences...),

- la gestion des écoulements aussi bien en lit mineur qu'en lit majeur (restauration et entretien des rivières, limitation du ruissellement. maintien des zones d'expansion de crues...).

Toutefois, elle veillera à ne pas empiéter sur les prérogatives du ministère de l'Environnement ou du ministère de l'Intérieur qui s'occupent de la gestion de la crise, ou encore sur ce qui relève du domaine de l'assurance c'est-àdire l'indemnisation des biens assurés.

Néanmoins, la mise en place de cette redevance n'a pas. à l'heure actuelle, reçu l'approbation du Premier Ministre (ni même des instances de l'Agence de l'Eau Seine-Normandie). Celui-ci a décidé en juin 96, de mettre en place pour le VII' Programme des Agences de l'Eau (I997-200I), un fonds de concours que verseraient les Agences au ministère de l'Environnement. Ce fonds serait « destiné à permettre l'attribution par l'Etat de subventions aux maîtres d'ouvrage locaux intervenant dans la restauration des cours d'eau et des champs naturels d'expansion des crues ». A suivre... 


\section{LES PERSPECTIVES}

L'amélioration qualitative et quantitative du réseau hydrographique et de ses espaces associés repose sur la prise en compte de leur fonctionnement; la satisfaction des usages en dépend. Cette nécessaire prise en compte repose sur quatre principes (rapport ASCA-1993) :

- la reconnaissance de la valeur des milieux associés et de leur indispensable connexion ;

- le respect de la dynamique hydraulique et morphologique des cours d'eau conditionnant la diversité des habitats, donc des communautés et de la capacité d'auto-épuration, d'où le concept d'espace de liberté ;

- le maintien de champs d'inondation fonctionnels pour une régulation naturelle des crues et pour l'importance que ces dernières présentent vis-à-vis des milieux ;

- une bonne gestion des nappes phréatiques sur le plan quantitatif et qualitatif.

En conséquence, il s'agit de faire une place aux infrastructures naturelles à côté des infrastructures artificielles, celles-ci constituant ensemble «les bases " de la gestion intégrée. Cette gestion ne concernerait plus un linéaire : le cours d'eau comme par le passé, mais un espace fluvial comprenant les lits mineurs et majeurs ainsi que les zones humides.

Cette logique sous-entend certaines évolutions nécessaires :

- la lutte contre la pollution doit intégrer les rejets d'origine agricole, ceux liés aux eaux pluviales, et les aspects eaux souterraines. Une meilleure connaissance de la fonction autoépuratrice doit contribuer à une meilleure gestion,

- la gestion de la ressource en eau appelle à la fois rigueur dans l'évaluation de l'augmentation de la demande, et effort en matière d'économie d'eau,

- l'aménagement et l'entretien des cours d'eau nécessitent une évaluation de l'impact de ces interventions sur les espaces concernés.

- la protection et la gestion des espaces naturels fluviaux font appel à la mise en place d'outils.

- les aménagements hydrauliques structurants dont on peut souhaiter :

- l'élargissement de l'étude des impacts,

- la mise en place d'une planification sociologique et écologique de l'aménagement des eaux,

- une prise de décision intégrant de manière plus importante les processus de concertation.

Je vais maintenant laisser place aux diverses voies proposées par plusieurs auteurs. Elles ne seront ici que résumées ; aussi je vous encourage, si vous ne l'avez déjà fait, à lire ces communications riches de pistes de réflexion, de retours d'expérience, d'axes de recherche...

H. Piegay - O. Barge - J.P. Bravard - N. Landon J.L. Peiry, dans leur communication intitulée «Comment délimiter l'espace de liberté des rivières ? ", nous présentent une démarche pour atteindre cet objectif. Celui-ci s'inscrit dans l'esprit de la loi sur l'eau de 1992 induisant la nécessité de principes de gestion territoriale pour contrôler l'implantation d'activités humaines dans cet espace potentiel de la rivière. Le SDAGE Rhône-Méditerranée-Corse définit la notion d'espace de liberté comme étant l'espace du lit majeur dans lequel le chenal ou les chenaux fluviaux assurent des translations latérales permettant une mobilisation des sédiments ainsi que le fonctionnement optimum des écosystèmes aquatiques et terrestres. Ces auteurs présentent une synthèse des travaux réalisés sur ce thème depuis 1990.

G. Barnaud, auteur de «Fonctions et rôles des zones humides » fait un point exhaustif sur ce que l'on doit savoir concernant ces espaces. Il s'agit tout d'abord de les définir et de les caractériser. Un bilan de leur « état de santé » a été dressé, déclenchant un Plan national d'actions pour leur sauvegarde. Mais celui-ci ne concernera pas toutes les zones humides. Leur atout réside dans le fait que gestion adéquate de la ressource en eau et bonne santé des zones humides forment un tandem qui devrait être compris par les collectivités locales dans leur choix en matière d'aménagement du territoire.

S. Laloux, auteur de « Promouvoir une gestion environnementale de l'espace rivière ", explique l'évolution de la problématique rivière pour tendre vers une prise en considération conjointe des trois axes Eau-Espace-Environnement. Une étude méthodologique a proposé, entre autres, la définition du concept de gestion des espaces rivières, une synthèse des outils d'intervention (techniques, économiques, administratifs), et l'analyse des structures maîtres d'ouvrage. L'approche des acteurs montre que la réponse technique n'est pas suffisante. Aussi, l'auteur propose-t-il des actions visant à faire évoluer les structures maîtres d'ouvrage.

F. Gross, dans sa communication intitulée « Berges et milieux riverains des cours d'eau : carrefour d'idées et conflits d'usages, quelles perspectives ? ", fait l'historique des aménagements de rivière montrant l'évolution des techniques et des mentalités. L'auteur dresse un bilan de l'entretien des cours d'eau domaniaux et non domaniaux et précise l'état des connaissances actuelles dans ce domaine. Puis, au travers des étapes de la réalisation d'un projet, des pistes de réflexion sont proposées, l'objectif recherché étant de pérenniser les travaux d'aménagement par de l'entretien régulier.

\section{QUESTIONS AUX AUTEURS}

1) H. Piegay - O. Barge - J.P. Bravard N. Landon - J.L. Peiry - UNMR 5600 « Environnement Ville Société » Lyon.

- Dans le cas du Giffre, vous avez donné une formule intéressante concernant la largeur de bande active qui peut servir de base à des calculs économiques. Même si la corrélation est significative, le coefficient de détermination est très modeste $(0,4)$, avez-vous une idée de I'intervalle de confiance de la largeur du corridor végétal nécessaire au bon fonctionnement de la bande de tressage ?

- A quel terme peut-on espérer des modèles, ou tout au moins des guides de détermination de l'espace de liberté ? Suffira-t-il de bases de données photographiques ? - Dans les exemples que vous donnez, y a-t-il eu concertation avec les riverains concernés ? Quelle a été leur réaction?

\section{Réponse à la question 1}

L'aléa "érosion " peut être apprécié non seulement d'un point de vue infocartographique mais aussi statistiquement. L'approche méthodologique succinctement présentée lors du colloque de la S.H.F. mars 96 a été expérimentée sur le Giffre à partir des missions photographiques de l'I.G.N. datant de 1934 et de 1984 (Piégay, sous presse). Un découpage du chenal en transects a été effectué afin de mesurer la largeur des différentes unités (bande active on 1934 ou 1984, zones érodées entre les deux dates). Cette analyse. réalisée sur 758 transects, a mis en évidence une relation statistique entre la largeur de la bande active et la largeur du lit majeur érodé. Un modèle linéaire a permis d'estimer que la largeur du corridor végétal nécessaire au bon fonctionnement de la bande de tressage représente environ $20 \%$ de la largeur de la bande active.

Deux points méritent d'être pourtant précisés : i) Le coefficient de détermination est effectivement faible mais néanmoins représentatif compte tenu de la valeur de $p$. Sa 
faiblesse s'explique par le fait que la population de transects qui a été considérée correspond à la fois à des secteurs instables et des secteurs stables. Si l'on ne s'en tient qu'aux secteurs instables, la largeur de l'espace tampon représente en fait $50 \%$ de la largeur de la bande active. Ce chiffre a par ailleurs été confirmé sur la rivière Drôme, le coefficient de détermination étant alors évidemment plus élevé $\left(r^{2}=\right.$ $0.91: p<0,0001)$ ii) Considérer toute la population de transects présente en fait un intérêt. En utilisant de tels modèles, nous pouvons estimer financièrement les terrains érodés à partir de la seule largeur de bande active et sans qu'il soit ainsi nécessaire d'identifier les zones effectivement érodées, ce travail étant en effet relativement lourd. Néanmoins, ces modèles ne permettent effectivement pas pour l'instant d'identifier les secteurs instables. Un travail est en cours actuellement pour savoir si la largeur des unités pionnières pourrait être un indicateur exploitable.

Piégay H., sous presse (1977). - «Principes nouveaux en matière de gestion des marges de rivières en tresses (exemple de la moyenne vallée de Giffre, Haute-Savoie, France) », Revue de Géographie Alpine.

\section{Réponse à la question 2}

Actuellement, nous disposons déjà d'un certain nombre d'outils méthodologiques permettant d'identifier "l'espace de liberté " des cours d'eau. Sous l'impulsion des S.D.A.G.E., notamment celui de R.M.C., l'espace de liberté devient une solution de gestion de l'érosion des berges complémentaire des solutions utilisées traditionnellement. En fait, ces différentes solutions ne sont pas forcément antinomiques mais devraient s'inscrire dans un plan de gestion de l'érosion des berges à l'échelle d'une rivière. Sur un tronçon donné, la plupart d'entre elles sont en effet nécessaires étant entendu que le risque d'érosion varie d'un secteur à l'autre (Piégay, 1995). Une étude, remise à l'agence de l'Eau R.M.C. en février 1996, pose les premiers principes méthodologiques de la définition de l'espace de liberté (Piégay et al., 1996a), elle avait un double objet : i) préciser les protocoles de détermination applicables sur les rivières en tresses, celles-ci présentant une dynamique latérale un peu différente de celle des rivières à méandres dont la connaissance était plus avancée, ii) établir une synthèse des différentes démarches conduites depuis 1990. Dès lors, une réflexion est aujourd'hui en cours dans le cadre de l'agence R.M.C., le but étant d'élaborer un guide technique de définition de l'espace de liberté à l'usage des maîtres d'auvre et des maîtres d'ouvrage.

Suffira-t-il de bases de données photographiques? La photographie serait-elle un Support si peu crédible pour que la question soit formulée ainsi? Les bases de données photographiques ont fait la preuve de leur pertinence dans de nombreux cas en matière de gestion de cours d'eau. Elles sont incontournables dans le cadre d'une détermination de l'espace de liberté fondée sur une tendance historique mais leur exploitation doit néanmoins se plier à un certain nombre de règles. De fait, trois démarches sont à l'heure actuelle possibles:

- La démarche fondée sur la tendance historique et l'exploitation des séries photographiques présente encore un potentiel de développement important. Des recherches sont actuellement en cours pour exploiter au mieux les modèles infocartographiques (Piégay et al., 1996b). Les tendances de déplacement mises en évidence par la superposition des tracés successifs pourraient servir de base à des modèles cartographiques de diffusion permettant d'identifier les espaces érodables à différents termes... compte tenu des tendances présentes.

- L'analyse statistique explicitée précédemment (question I) est également prometteuse. Son avantage réside dans sa simplicité puisqu'elle n'est fondée que sur un seul chiffre, la largeur de l'espace tampon à considérer. Sur les rivières à méandres, je crois qu'il est actuellement possible de définir également l'espace de liberté à partir de modèles hydrauliques, de l'analyse de la variabilité tridimensionnelle de la géométrie du lit. La littérature internationale est riche et des outils existants peuvent être adaptés à notre problème, encore faut-il trouver des spécialistes pour s'y intéresser. La rivière en tresses semble par contre plus difficile à appréhender.

Piégay H., 1995, «Compte rendu de la mission d'expertise "Gestion des vallées alluviales ", rapport final ", CEMAGREF et Agence de I'Eau R.M.C..

Piégay H., Landon N., Barge O. Citterio A.. 1996a. «Contribution à la définition d'une méthode de la détermination de l'espace de liberté », rapport, CNRS, Agence de l'eau R.M.C..

Piégay H., Barge O., Landon N.. 1996. - «Steamway concept applied to river mobility/human use conflict management ", Colloque de I'International Water Resources Association : "Rivertech'96: new/emerging concept for rivers », Chicago, Etats-Unis, Sept. 96.

\section{Réponse à la question 3}

La réussite d'un projet " espace de liberté », sur les rivières non domaniales notamment, ne dépend pas uniquement de notre savoir-faire technique. Le problème foncier et par conséquence humain est au caur de la démarche. Définir puis gérer un espace de liberté ne peut se concevoir qu'avec le soutien actif des collectivités locales sachant que cette démarche repose non seulement sur une logique patrimoniale mais surtout sur une logique économique. Les approches conduites sur l'Ain et la Galaure (Bravard et al, 1990 ; Cuaz et al., 1996) ont démontré que laisser divaguer la rivière sur certains secteurs était financièrement la meilleure solution de gestion. Le problème auquel le technicien est alors confronté est affectif. Il y a un attachement à la terre. Plusieurs enquêtes réalisées auprès des propriétaires riverains ont montré que ceux-ci étaient fortement attachés à leur bien au point de reconnaître qu'il n'a aucune valeur et que pourtant ils souhaitent continuer à le posséder. voire à le préserver de l'instabilité de la rivière (Piégay et Lama, 1995). Un autre problème est celui de l'anthropocentrisme latent issu du lien ancestral que l'on a tissé avec notre environnement. Notre relation à la rivière ne se conçoit bien souvent qu'à travers sa maîtrise. Nous sommes encore peu capables aujourd'hui de gérer, de nous adapter à l'instabilité naturelle: la trame foncière par exemple est rigide et donc totalement inadaptée aux changements $d u$ milieu. La volonté d'aménager n'est donc pas forcément économique mais repose sur la tradition. La pression locale poussant à l'aménagement est d'autant plus vive que les hommes de la vallée ont traditionnellement participé à la maîtrise de la rivière. Définir un espace de liberté est ainsi une solution rejetée par bien des personnes sur les rivières intra-alpines alors qu'elle est envisagée par les riverains sur les rivières de piémont (Ain, Ardèche, Galaure), leurs ancêtres s'étant plus facilement adaptés à l'instabilité de la rivière compte tenu d'une plus grande disponibilité de terres.

Définir un espace de liberté apparaît une solution patrimoniale et financière adaptée à une gestion durable et équilibrée usages-milieu. Elle ne peut réussir que si les riverains sont amenés à participer au projet et à réfléchir collectivement sur la démarche, l'intérêt de celle-ci apparaissant progressivement. Par ailleurs, les gestionnaires ont pris conscience de la nécessité de promouvoir une telle démarche ; une volonté collective et des outils financiers et administratifs existent aujourd'hui pour la mener à bien.

Bravard J.-P., Franc O., Landon N., Large J.L. et Peiry J.L., 1990. - La basse vallée de l'Ain : étude géomorphologique, PIREN-CNRS. Agence de l'Eau R.M.C., 113 p. 
Cuaz M., Javelle E., Piégay H., Mandier P., 1995. «Etude pilote de définition et d'analyse de la bande de méandrage de la rivière Galaure sur les plans dynamique et foncier entre la limite du département de I'Isère et l'Ouest de Saint-Uze». Syndicat Intercommunal du Bassin de la Galaure et Agence de l'Eau R.M.C..

Piégay H. et Lama E., 1995. - « L'intégration des stratégies foncières individuelles dans la gestion des lits majeurs boisés (exemple de quatre rivières de rang intermédiaire du bassin du Rhône) ». Revue de Géographie Alpine, 1 89-110.

2) G. Barnaud - Museum d'Histoire Naturelle " Rôle et fonction des zones humides $»$.

- Pouvez-vous nous expliquer la notion d'infrastructure naturelle? Quelles en sont les implications juridiques et administratives ?

Pour préserver les zones humides (marais, marécage, tourbière, vasière), les protecteurs de la nature ont développé, à la fin des années soixante, un argumentaire fondé sur leur grand intérêt écologique, floristique et faunistique. A titre indicatif, alors que les zones humides occupent seulement $5 \%$ environ de la superficie des continents, elles abritent 35 $\%$ des espèces rares et en danger à l'échelon mondial. Il est d'ailleurs significatif que ces milieux soient les seuls bénéficiant, dès 1971. d'une convention internationale visant à leur conservation, celle de Ramsar (Convention relative aux zones humides d'importance internationale particulièrement comme habitats des oiseaux d'eau), ratifiée par 95 pays dont la France.

Au cours des années soixante-dix, tirant les leçons des effets constatés de la destruction de zones humides, des experts ont mis en évidence la variété de leurs rôles écologiques et environnementaux. Les termes de fonctions écologiques et de valeurs des zones humides ont alors été intégrés au discours des promoteurs de la conservation de ces milieux. Les fonctions sont déduites directement de leurs caractéristiques et fonctionnements écologiques, les valeurs ou services rendus étant estimés par les avantages économiques et culturels (exploitation des ressources agricoles, halieutiques, cynégétiques ; régulation des régimes hydrologiques et de la qualité de l'eau : loisirs...) retirés par les populations locales et plus largement par la société.

Ce type d'approche a été développé par l'OCDE pour étudier les défaillances du marché et des gouvernements dans la gestion de l'environnement et plus particulièrement des zones humides. Les fonctions et valeurs par grands types de zones humides ont ainsi été identifiés [I].

Ces travaux et d'autres ont permis de cerner le rôle des zones humides vis-à-vis :

- du contrôle des inondations par le stockage d'une partie de l'eau, et l'" écrêtage » des pics de crue :

- du soutien des débits d'étiage par "relargage "différé de l'eau stockée au moment des crues :

- de l'épuration physique (rétention des matières en suspension) et chimiques de l'eau (dénitrification, recyclage du phosphore, piégeage de métaux lourds et de micropolluants organiques):

- de la recharge des nappes phréatiques dans certaines circonstances ;

- de la stabilisation des berges et rives, de la lutte contre l'érosion:

- de la production d'espèces végétales (roseaux, bois, fourrage) et animales, en particulier halieutiques et cynégétiques

- du maintien de la biodiversité, en France, $50 \%$ de l'avifaune et $30 \%$ des espèces végétales remarquables et menacées sont inféodés aux zones humides :

- de la qualité paysagère de certaines régions :

- de l'évolution des microclimats...

Dans cette logique de prise en compte élargie de la place et du rôle des zones humides dans un territoire donné, un groupe d'experts qui réfléchissait sur des propositions innovantes pour la gestion des fleuves dans le cadre de la Loi sur l'eau, a pour la première fois formulé la notion d'insfrastructure naturelle afin de "rétablir l'équilibre entre le volet naturel et le volet artificiel » de la gestion de l'eau [2].

Tableau 1. - Mise en parallèle des fonctions et valeurs hydrologiques de zones humides, des effets de leur destruction ou dégradation, et des moyens nécessaires à la réparation-compensation de certains dommages occasionnés.

\begin{tabular}{|c|c|c|}
\hline Fonctions et valeurs & $\begin{array}{l}\text { Effets des destructions ou } \\
\text { dégradations }\end{array}$ & $\begin{array}{l}\text { Équipements et mesures } \\
\text { de substitution }\end{array}$ \\
\hline $\begin{array}{l}\text { - Action tampon vis-à-vis des } \\
\text { crues, retardement de la propaga- } \\
\text { tion des flux }\end{array}$ & - Inondation & $\begin{array}{l}\text { - Construction de barrages-écrê- } \\
\text { teurs, endiguements... } \\
\text { - Remboursement des dégâts }\end{array}$ \\
\hline $\begin{array}{l}\text { - Stockage des eaux et recharge } \\
\text { des nappes phréatiques }\end{array}$ & $\begin{array}{l}\text { - Sécheresse (augmentation de } \\
\text { l'intensité et de la durée) }\end{array}$ & $\begin{array}{l}\text { - Construction de barrages-réser- } \\
\text { voirs } \\
\text { - Creusement de puits plus pro- } \\
\text { fonds, captage d'eau à distance et } \\
\text { transport }\end{array}$ \\
\hline $\begin{array}{l}\text { - Stockage des effluents naturels } \\
\text { ou artificiels, élimination/transfor- } \\
\text { mation des nutriments et épuration } \\
\text { des eaux pollués }\end{array}$ & $\begin{array}{l}\text { - Augmentation des taux de pollu- } \\
\text { tion, phénomènes d'eutrophisation } \\
\text { - Risque toxicologique (ostréiculture) }\end{array}$ & $\begin{array}{l}\text { - Installation de stations d'épura- } \\
\text { tion des eaux usées } \\
- \text { Mise en place de systèmes } \\
\text { d'oxygénation des cours d'eau } \\
\text { - Construction de bassins de } \\
\text { contrôle sanitaire }\end{array}$ \\
\hline $\begin{array}{l}\text { - Stabilisation des sédiments, pro- } \\
\text { tection des rivages/érosion/tempêtes }\end{array}$ & $\begin{array}{l}\text { - Déstabilisation et érosion des } \\
\text { côtes, berges et lits de rivière } \\
\text { - Comblement des lacs, étangs, } \\
\text { baies et ouvrages hydrauliques } \\
\text { (canaux, barrages, réservoirs...) } \\
\text { - Augmentation des bouchons } \\
\text { vaseux }\end{array}$ & $\begin{array}{l}\text { - Enrochement, construction d'épis } \\
\text { - Construction de bassins de } \\
\text { décantation, de seuils, de passes à } \\
\text { poissons, renforcement des piles de } \\
\text { ponts } \\
- \text { Désenvasement de lacs, étangs } \\
\text { et estuaires }\end{array}$ \\
\hline
\end{tabular}


Reprise officiellement par l'Instance d'évaluation des politiques publiques [3]. cette notion a été affichée dans le Plan d'action pour les zones humides pour tenter de faire reconnaître le double bénéfice fonctionnel et patrimonial fourni par les zones humides. Dans le Plan, il est spécifié que la démonstration de l'intérêt écologique. économique et sociologique de la conservation des zones humides conduit maintenant à les "considérer comme des infrastructures naturelles qui devront être prises en compte en tant que telles dans les politiques d'aménagement du territoire. de modernisation agricole, de tourisme et de gestion de l'eau ".

Depuis, le concept a été structuré en partant du principe que l'élimination de tel type de fonction ou valeur par la destruction d'une zone humide nécessite la mise en place d'équipements artificiels ou de mesures de compensation (tab. 1) 14. 51. Or ces aménagements se caractérisent par des coûts d'investissement et d'entretien sans commune mesure avec les budgets affectés à la gestion des zones humides, et sans garantie d'un fonctionnement correct sur le long terme. Par exemple, le remplacement par un barrage d'écrêtement des crues, de l'infrastructure naturelle que représente la zone inondable de la Bassée entre Nogent-surSeine et Bray-sur-Seine, a été estimé entre 650 millions et 2 milliards de francs. Le traitement de l'eau potable de la nappe située sous les zones humides de la vallée de la Saône, rendu nécessaire par la progression du retournement des prairies humides et de leur mise en culture intensive (maïs) à proximité de nombreux champs captants, a été évalué entre 30 et 72 millions de francs par an.

Certains écologues et naturalistes rejettent l'utilisation de ce concept en invoquant : (I) la valeur patrimoniale intrinsèque de ces milieux, 2) le danger à les faire considérer comme des équipements au service du développement du territoire. (3) le risque représenté par ce parti pris « utilitariste ". En effet, il n'est pas toujours possible actuellement d'estimer financièrement la valeur réelle de tous les types de zones humides. De plus, les marais rendent souvent simultanément plusieurs services alors que les infrastructures artificielles ont en général une vocation unique.

Dans le but de modifier la perception encore largement négative de ces milieux par divers cercles influents (corps techniques, élus, grands publics), on a décidé au Séminaire "Quelle politique publique pour les zones humides francaises?" (Carentan. 27-29/10/1994 [6]) de divulguer la notion d'infrastructure naturelle afin de faire comprendre les fonctions écologiques et valeurs économiques des zones humides pour qu'elles soient considérées comme de véritables instruments de l'aménagement du territoire.

Pour maintenir ces infrastructures naturelles, le Plan d'action pour les zones humides prévoit la mise en cohérence des politiques publiques par:

- un recensement des mesures défavorables dans les textes législatifs et réglementaires, et leur révision:

- la prise en compte des charges liées à leur gestion et protection dans le cadre de la dotation aux collectivités locales: la réduction de la fiscalité sur le foncier non bâti. la suppression des taxes foncières (étangs, lacs), la réduction de la part communale: une orientation des mesures agri-environnementales et du Fonds de gestion de l'espace rural en leur faveur: un soutien à l'élevage extensif ; la suppression des aides publiques aux travaux d'aménagement agricole ou forestier:

- leur inscription aux POS : l'évaluation des conséquences économiques de leur altération : la reconstitution de milieux de mêmes fonctionnalités par les maîtres d'ouvrage de projets d'aménagement soumis à autorisation: la prise en compte de la préservation du fonctionnement des zones humides d'intérêt national lors de leur traversée par des voies routières ou ferroviaires.

S'il est réellement admis et mis en auvre par l'ensemble des partenaires concernés, le changement de cap et d'échelle engendré par le Plan d'action devrait aboutir à une stabilisation des destructions et dégradations et, peutêtre, à la reconquête de zones humides. Or la gestion de la ressource en eau, en grande partie conditionnée par le maintien en bonne santé et l'entretien des zones humides restantes à l'échelon des bassins versants, correspond à l'un des défis majeurs à relever au XxI siècle.

[1] TURNER R.K., 1992. - Défaillances des politiques dans la gestion des zones humides. Pages 9-17 in Les défaillances du marché et des gouvernements dans la gestion de l'environne ment. Les zones humides et les forêts, OCDE, Paris.

|2| Mermet L. (éd.), 1984. - Innover pour une gestion plus écologique des fleuves. Rapport du groupe de travail sur l'innovation en matière d'aménagement et de gestion de l'espace fluvial. AScA. Ministère de l'Environnement.

|3| Bernard P. (éd.), 1994. - Les zones humides. Comité interministériel de l'évaluation des politiques publiques. Premier Ministre. Commissariat au Plan. Rapport d'évaluation. La Documentation Française, 391 p.

[4] Mermet L., 1995. - Les infrastructures naturelles: statut, principe, concept, ou slogan? ZH Infos, 7: 7-9.

15] Lefebvre Ch., Barnaud G.. 1996. - Le concept d'infrastructure naturelle. Le cas des zones humides. Note rédigée pour le Comité national Ramsar. $6^{\text {e }}$ Session de la Conférence des Parties contractantes, Brisbane (Australie), 19-27 mars 1996, $2 \mathrm{p}$

[6] AnONYme, 1994. - Quelle politique publique pour les zones humides françaises? Vers un plan décennal d'utilisation avisée. Actes du séminaire sur les zones humides tenu à Carentan, les 27-29 octobre 1994. Ministère de l'Environnement/PNR du Marais du Cotentin et du Bessin, 209, les 27-29 octobre 1994. Ministère de l'Environnement/PNR du Marais du Cotentin et du Bessin, $209 \mathrm{p}$.

3) F. Gross - Rivière-Environnement.

"Les berges de cours d'eau : carrefour d'idées et conflits d'usage. Quelles perspectives?».

- Pérenniser l'aménagement par de l'entretien régulier, tel est le but recherché. Quels sont les obstacles à l'entretien des cours d'eau ?

- Pouvez-vous nous exposer quelques pistes de réflexion visant à faciliter la tâche des maîtres d'ouvrage et à créer une dynamique dans ce domaine ?

\section{Réponse à la question 1}

Ces obstacles sont de trois ordres, l'obstacle majeur étant d'ordre financier : en milieu rural, les collectivités locales. qui bien souvent se sont endettées lourdement pour faire réaliser des travaux de restauration, n'ont pas toujours les fonds nécessaires pour se lancer dans un programme pluriannuel d'entretien. Le deuxième obstacle est plutôt lié à la technique: quelles sont les tâches nécessaires et suffisantes pour réaliser un entretien valable en fonction des usages. qui soit également respectueux des potentialités de l'écosystème rivière. Par ailleurs, le marché n'est pas encore très porteur pour les entreprises; on ne trouve pas toujours la structure adéquate pour faire ce type de travail.

\section{Réponse à la question 2}

Il serait souhaitable que les maîtres d'ouvrage puissent bénéficier de conseils techniques et trouvent une écoute lorsqu'ils sont perplexes devant la façon de monter un dossier de demande de financement, par exemple. Pour cela, la création de cellule au niveau départemental me paraît indispensable: appelés, selon les départements CATER (Cellule 
d'Assistance Technique à l'Entretien des Rivières) ou SATERCE (Service d'Assistance Technique à l'Entretien Régulier des Cours d'Eau), un certain nombre d'organismes de ce type fonctionnent déjà. Des techniciens sont mis à la disposition des collectivités locales et réalisent, en particulier : élaboration d'un programme pluri-annuel d'entretien, recherche des financements, surveillance de chantier.

Mais il serait également très souhaitable qu'au niveau des syndicats de communes, il y ait embauche de technicienrivière. On revient donc à l'aspect financier.

\section{VII $\square$ CONCLUSION}

Pour avoir trop longtemps géré flux hydrauliques et flux polluants en négligeant leurs lieux de transit, c'est-à-dire les divers milieux aquatiques et leurs peuplements, des situations de déséquilibre ont vu le jour. Elles ne peuvent qu'inciter à une gestion globale de l'espace fluvial, et pas seulement du cours d'eau. Ce changement d'approche a été largement exposé et appuyé par les communications de la question II. Si la réponse technique a une grande importance dans les progrès à venir, la prise en compte des acteurs, de leurs attentes et les réponses que nous saurons leur apporter conditionneront l'avenir dans cette étape transitoire.

Elle nécessitera :

- afin de mieux connaître, pour mieux agir, un développement de la recherche dans la connaissance du fonctionnement des systèmes fluviaux, ainsi que la réalisation d'études d'aide à la décision,

- I'élaboration d'indicateurs de synthèse permettant de suivre, entre autres :

- l'impact des usages sur les écosystèmes,

- le degré de satisfaction des usages,

- l'impact des pollutions sur la santé et l'environnement,

- une mobilisation des acteurs économiques et de la recherche pour développer des activités moins polluantes et moins consommatrices d'eau,

- une mobilisation des acteurs techniques, administratifs et financiers pour pratiquer l'interdiscipline autour des projets,

- une sensibilisation par l'information et la formation,

- un glissement de l'approche environnementale au concept de développement durable qui vise à satisfaire les besoins présents à condition de ne pas compromettre la satisfaction des besoins des générations futures. 